\title{
THROES: a caTalogue of HeRschel Observations of Evolved Stars
}

\section{PACS range spectroscopy ${ }^{\star}$}

\author{
J. Ramos-Medina ${ }^{1}$, C. Sánchez Contreras ${ }^{1}$, P. García-Lario ${ }^{2}$, C. Rodrigo ${ }^{1}$, J. da Silva Santos ${ }^{1}$, and E. Solano ${ }^{1}$ \\ 1 Department of Astrophysics, Astrobiology Center (CSIC-INTA), ESAC campus, PO Box 78, 28691 Villanueva de la Cañada, \\ Madrid, Spain \\ e-mail: jramos@cab.inta-csic.es \\ 2 European Space Astronomy Centre, European Space Agency, PO Box 78, 28691 Villanueva de la Cañada, Madrid, Spain
}

Received 13 September 2017 / Accepted 27 October 2017

\begin{abstract}
This is the first of a series of papers presenting the THROES (A caTalogue of HeRschel Observations of Evolved Stars) project, intended to provide a comprehensive overview of the spectroscopic results obtained in the far-infrared $(55-670 \mu \mathrm{m})$ with the Herschel space observatory on low-to-intermediate mass evolved stars in our Galaxy. Here we introduce the catalogue of interactively reprocessed Photoconductor Array Camera and Spectrometer (PACS) spectra covering the 55-200 $\mu \mathrm{m}$ range for 114 stars in this category for which PACS range spectroscopic data is available in the Herschel Science Archive (HSA). Our sample includes objects spanning a range of evolutionary stages, from the asymptotic giant branch to the planetary nebula phase, displaying a wide variety of chemical and physical properties. The THROES/PACS catalogue is accessible via a dedicated web-based interface and includes not only the science-ready Herschel spectroscopic data for each source, but also complementary photometric and spectroscopic data from other infrared observatories, namely IRAS, ISO, or AKARI, at overlapping wavelengths. Our goal is to create a legacy-value Herschel dataset that can be used by the scientific community in the future to deepen our knowledge and understanding of these latest stages of the evolution of low-to-intermediate mass stars.
\end{abstract}

Key words. catalogs - stars: evolution - stars: AGB and post-AGB - infrared: stars

\section{Introduction}

Herschel $^{1}$ (Pilbratt et al. 2010), launched in May 2009, has provided a new vision of the whole Universe in the far infrared (FIR) thanks to the capabilities of the three instruments on board: the Heterodyne Instrument for the Far-Infrared (HIFI; de Graauw et al. 2010), Spectral and Photometric Imaging Receiver (SPIRE; Griffin et al. 2010) and the Photoconductor Array Camera and Spectrometer (PACS; Poglitsch et al. 2010). In particular, Herschel has been extremely useful in the study of the complex physical and chemical processes that take place in the final stages of stellar evolution. For example, observations from the Mass-loss of Evolved Stars (MESS) observing programme (Groenewegen et al. 2011) have demonstrated the capability of Herschel to image the circumstellar envelopes (CSEs) of evolved stars and the interaction regions between the stellar winds and the interstellar medium with unprecedented detail. In the spectroscopic area, studies have addressed the analysis of the circumstellar forsterite (e.g. de Vries et al. 2011) and the CO line emission (e.g. De Beck et al. 2010; Danilovich et al. 2015; Maercker et al. 2016). The detection of rotational emission lines of $\mathrm{OH}^{+}$for the first time in three planetary nebulae $(\mathrm{PNe})$ (Aleman et al. 2014) with observations taken as part of the HerPlans observing programme (Ueta et al. 2014), or the detection

\footnotetext{
$\star$ The THROES/PACS catalogue is accessible at https://throes. cab.inta-csic.es/

1 Herschel is an ESA space observatory with science instruments provided by European-led Principal Investigator consortia and with important participation from NASA
}

of warm water vapour around IRC +10216 (Decin et al. 2010), the closest C-rich star to our solar system, are also good examples of the contribution of Herschel to our understanding of the chemical complexity of these CSEs and can also be considered as important highlights of the mission in the field.

Towards the end of their lives, low-to-intermediate mass stars (1 $M_{\odot} \leq M \leq 8 M_{\odot}$ ) have burnt up their central hydrogen and helium, leaving a quiescent $\mathrm{C}-\mathrm{O}$ core with $\mathrm{H}$ and $\mathrm{He}$ fusion reactions taking place in thin shells surrounding the inner core. These objects are ascending the asymptotic giant branch (AGB), a phase of stellar evolution during which their atmospheres expand and cool down, characterized by an intense mass loss (from $10^{-7}$ to $10^{-4} M_{\odot} \mathrm{yr}^{-1}$ ) that results in the formation of a CSE of gas and dust around the central star, which emits very strongly in the mid-to far-infrared wavelengths (Habing 1996). Once the star terminates the AGB phase, the mass-loss rate suddenly decreases and the temperature of the central object becomes, progressively, high enough to induce the onset of the ionization of the gas in the surrounding CSE. If the temperature increases on a timescale shorter than the dispersion time of the matter previously ejected, we will observe an ionized planetary nebula (PN) (Kwok 2005).

The intermediate stage between the AGB and PN phases is called the post-AGB phase. This phase is also known as pre-PN phase, although it is not clear whether all post-AGB stars will develop a PN. During the post-AGB phase, the shell, formed in the AGB phase, detaches from the central star, the spherical symmetry is broken, and fast bipolar or multi-polar winds appear (see e.g. Balick \& Frank 2002, for a review). This is a very 
short-lived phase ( $\sim 1000$ years) and, as a consequence of it, a not very well understood stage of stellar evolution.

The infrared and sub-millimetre regions offer a rich variety of diagnostic, atomic, ionic, molecular, and solid-state spectral features, and are particularly well suited to study the complex physical and chemical properties found in AGBs, post-AGBs, and $\mathrm{PNe}$, which may be very different from source to source, depending on critical parameters like the initial mass or the metallicity. A large sample of low- and intermediate-mass evolved stars in our Galaxy were observed by the PACS instrument onboard Herschel under many different observing programmes, and the associated automatically pipeline-processed data are now publicly available from the Herschel Science Archive ${ }^{2}$ (HSA) after the end of the proprietary period of one year. In the vast majority of cases, unfortunately, the PACS spectroscopy pipeline products in the HSA cannot be considered scienceready, and they would strongly benefit from dedicated interactive data reduction to help remove the residual instrument artefacts and to improve the absolute flux calibration as a necessary further step in order to become publication-quality products.

With this aim, we have interactively processed in a systematic and homogeneous way all PACS range spectroscopic observations contained in the HSA corresponding to stars that can be identified as low- or intermediate-mass evolved stars, with the exception of a small subset of nearby sources that show very extended emission and a few cases where the unchopped observing mode was used, as they require a special case-by-case treatment, which is beyond the scope of this project. The result of this interactive data reduction effort has been used to compile the first version of THROES: (a caTalogue of HeRschel Observations of Evolved Stars), through which our final data products are made available to the community for scientific exploitation. We are currently working on a second version of the THROES catalogue that will also incorporate spectroscopic data from the Herschel/SPIRE instrument (Ramos Medina et al., in prep.).

This paper is organized as follows. In Sect. 2 we describe how the observations were performed, the building of the THROES sample, and the main characteristics of the sources included in it. In Sect. 3 the main data reduction steps applied are described. In Sect. 4, we introduce the contents of the THROES catalogue and its web interface. In Sect. 5, we try to characterize the quality of our science data products through comparisons with the standard pipeline products contained in the HSA and with observations taken by other space-based facilities in the past, like IRAS, AKARI, and ISO. The final summary is given in Sect. 6.

\section{Observations}

\subsection{PACS spectroscopy}

\subsubsection{The PACS spectrometer}

The PACS spectrometer covers nominally the wavelength range from 51 to $210 \mu \mathrm{m}$ in two different channels that operate simultaneously in the so-called blue (51 to $105 \mu \mathrm{m})$ and red (102 to $220 \mu \mathrm{m}$ ) bands. The field of view (FoV) covers a $47^{\prime \prime} \times 47^{\prime \prime}$ region in the sky using an array composed of $5 \times 5$ square spatial pixels (hereinafter "spaxels"), each one 9.4 " $\times 9.4$ " in size, with 16 pixels along the spectral dimension that are shifted to sample the whole wavelength range to be covered. PACS provides a resolving power between 940 and 5500 (i.e. a spectral resolution of 75 to $300 \mathrm{~km} \mathrm{~s}^{-1}$ ) depending on the wavelength range. As

\footnotetext{
2 http://archives.esac.esa.int/hsa/whsa
}

shown in the PACS Observer's Manual ${ }^{3}$, the PSF (point spread function) of the PACS spectrometer ranges from $\sim 9^{\prime \prime}$ in the blue band to $\sim 14^{\prime \prime}$ in the red band.

\subsubsection{Astronomical observing templates (AOTs)}

Two different observation schemes or AOTs (astronomical observing templates) were offered to the users of the PACS spectrometer. The LineScan Mode was intended for the observation of one or a limited number of narrow spectral line features, while the RangeScan Mode was optimized for the observation of broad spectral lines or features, including the possibility to observe the full spectral range of the selected orders in "SED (Spectral Energy Distribution) mode". Both configurations generate data in a $3 \mathrm{D}$ cube format (flux versus wavelength and spatial position).

\subsubsection{Observing modes}

For each of the AOTs above described, PACS also offered two different observing modes: "Standard chopping-nodding mode" (hereafter "Chop/Nod") and "Unchopped grating scan mode" (hereafter "Unchopped"); the difference between these two modes lies in the observing technique used to allow the telescope and astronomical background to be subtracted from the signal coming from the source. "Chop/Nod" observations point, alternatively, to the On- and Off-positions and collect the Onand Off-data in a common "ObsID". The "Unchopped" ones take one observation for the On-source position and another different observation for the Off-source pointing; they are then processed independently. After that, the user has to carry out the required On-Off subtraction.

\subsubsection{Pointing modes}

Every observation was also defined by the pointing mode, which could be "pointed" using a single pointing on the source; or "mapping", a composition of different pointings that, combined, were used to generate maps with improved beam sampling and a larger FoV. More information about the different instrument AOTs, observing, and pointing modes can be found in the PACS Observer's Manual.

\subsection{Building the THROES sample}

Herschel successfully performed more than 37000 science observations during its operational lifetime. The full list can be found in the Herschel Observing $\log ^{4}$. It contains a total of 530 PACS spectroscopy observations executed successfully, associated to 44 science proposals that were submitted under the "Evolved Stars/Planetary Nebulae/Supernova Remnants" category by the original proposers. Out of these original $530 \mathrm{ob}-$ servations, 347 were identified as corresponding to low- or intermediate-mass stars, according to the information available in the bibliography, of which a subset of 258 were taken in PACS RangeScan mode.

All these observations were originally included in the THROES sample. However, in the final version of the catalogue, we discarded 7 observations of a small group of extended, nearby sources taken in PACS "mapping" mode, as they would require a dedicated reprocessing adapted to the specific characteristics of each source, an effort that in most cases has been

\footnotetext{
herschel.esac. esa.int/Docs/PACS/pdf/pacs_om.pdf

4 Available at http://herschel.esac.esa.int/obslog/
} 
or is being carried out by the research groups that requested the original observations. Similarly, we excluded 20 additional observations taken in "Unchopped" mode from our reprocessing as these are in general more complex observations affected by technical problems that would deserve a special case-by-case treatment, beyond the scope of this project. Finally, 11 observations failed during the reprocessing for different reasons and were not included in this first version of the THROES catalogue. In particular, one observation of the planetary nebula NGC 6153 (ObsID 1342249998) and another one of NGC 7662 (ObsID 1342246642) both failed because of the too narrow spectral range covered, centred at the forbidden [O III] emission line at $52 \mu \mathrm{m}$, at the edge of the spectral coverage of the PACS blue detector where the spectral response function is not well characterized; six observations (ObsIDs: 1342230895 and 1342230905 to 1342230909 ) associated to the Red Rectangle failed because they cover very narrow spectral regions affected by leakage; an observation of the post-AGB star HR 4049 (ObsID 1342247550), covering a very short wavelength region between 103 and $116 \mu \mathrm{m}$, was extremely noisy in the red channel and failed reprocessing in the blue channel; and finally, we could not reprocess two very long exposure SED mode observations of the proto-planetary nebula IRAS $01005+7910$ (ObsIDs 1342247005 and 1342247006) as they demanded too much memory, exceeding the capacity of our local hardware environment. In summary, a total of 220 Herschel observations (ObsIDs) were finally considered for interactive data reduction, corresponding to 114 individual targets (see full list in Table A.1), comprising a total of 440 individual spectral ranges.

\subsection{Characteristics of the THROES sample}

\subsubsection{Evolutionary stage and IRAS colours}

Information on the evolutionary stage of each object in the THROES sample was extracted from the SIMBAD (Set of Indications, Measurements, and Bibliography for Astronomical Data) database and from the literature. Accordingly, we have classified our objects into four main groups: AGB stars, OH/IR stars (extreme O-rich AGB stars with high mass-loss rates and long variability periods), post-AGB stars (or pre-PNe), and PNe. In its current version, the catalogue contains PACS range spectra for $43(38 \%)$ AGB stars, $17(15 \%)$ OH/IR stars, $29(25 \%)$ post-AGB stars, and 25 (22\%) PNe (see Fig. 1).

Figure 2 shows the distribution of our targets in the IRAS two-colour diagram, where the location of various types of sources is indicated, following the original description presented by van der Veen \& Habing (1988). This diagram illustrates the variety of evolutionary stages covered by the THROES sample. The AGB stars are distributed along a sequence of increasing infrared excess, which represents the evolution expected during the AGB as a result of the formation of thick and dense shells of dust and gas around these mass-losing stars, with the reddest IRAS colours corresponding to the most extreme OH/IR stars. Once the mass-loss phase ends, objects evolve towards the right in the two-colour diagram (region IV, V, and VIII in the plot), which are the areas populated by most of the sources in our sample classified as post-AGB stars and PNe, surrounded by detached cool dust shells.

\subsubsection{Galactic distribution}

The galactic distribution of the THROES sample is shown in Fig. 3. Our sources are strongly concentrated at relatively low

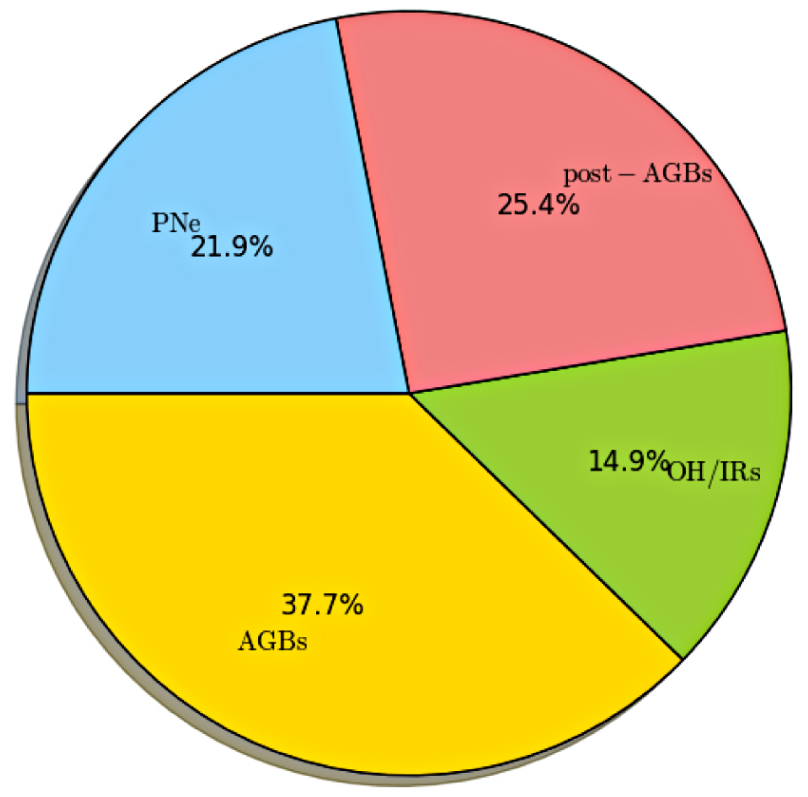

Fig. 1. Pie chart illustrating the distribution of stars in the THROES sample according to their evolutionary stage.

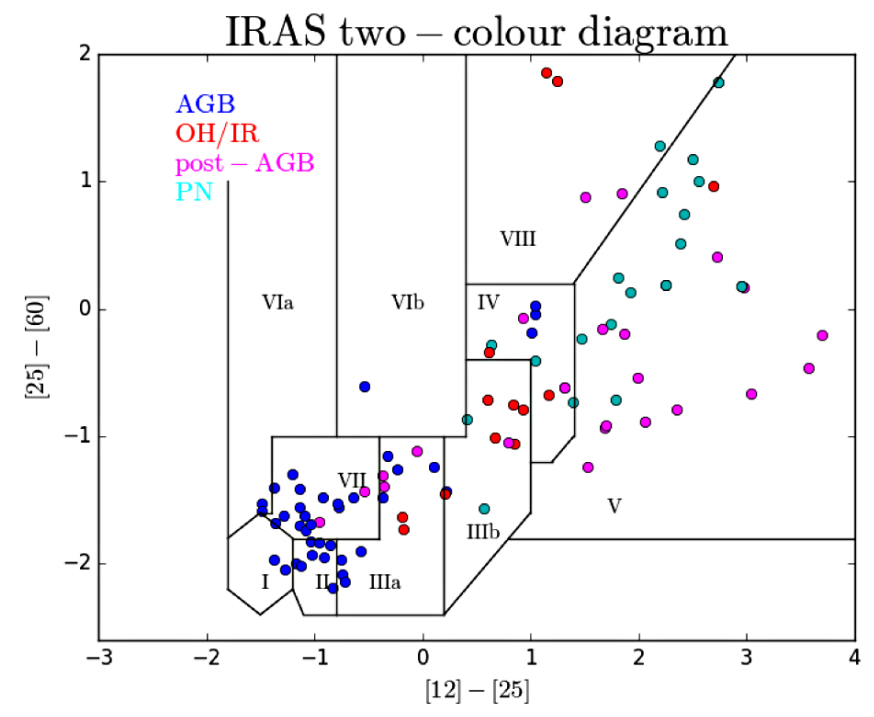

Fig. 2. IRAS colour-colour diagram of the THROES sample with good quality IRAS data (Quality Flag $=3$ ) in the 12,25 , and $60 \mu \mathrm{m}$ bands. The diagram is divided into different boxes where sources with different characteristics and evolutionary stage are contained (see van der Veen $\&$ Habing 1988, for a detailed description).

galactic latitudes, as expected from a distant population of luminous sources concentrated in the galactic disk, although a significant number of them are also observed at high galactic latitudes, corresponding to the small fraction of bright, nearby sources. Interestingly, most of the $\mathrm{OH} / \mathrm{IR}$ stars are found at very low galactic latitudes, as they correspond to a population of stars that are proposed to represent the most massive precursors of $\mathrm{PNe}$ (García-Hernández et al. 2007).

\subsubsection{Dominant chemistry}

Asymptotic giant branch stars are generally classified as O-rich (M-type) or C-rich (C-type) based on the $\mathrm{C} / \mathrm{O}$ ratio found in their outer envelope. Objects with $\mathrm{C} / \mathrm{O}>1$ are considered $\mathrm{C}$-rich stars 

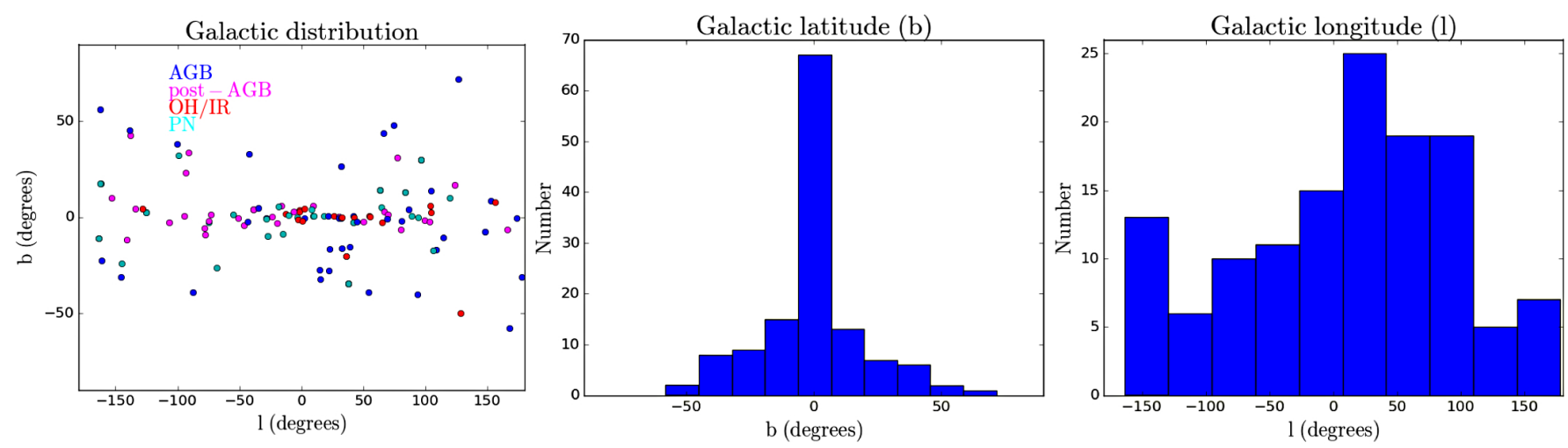

Fig. 3. Galactic distribution of the THROES targets. Colour code as in Fig. 2.

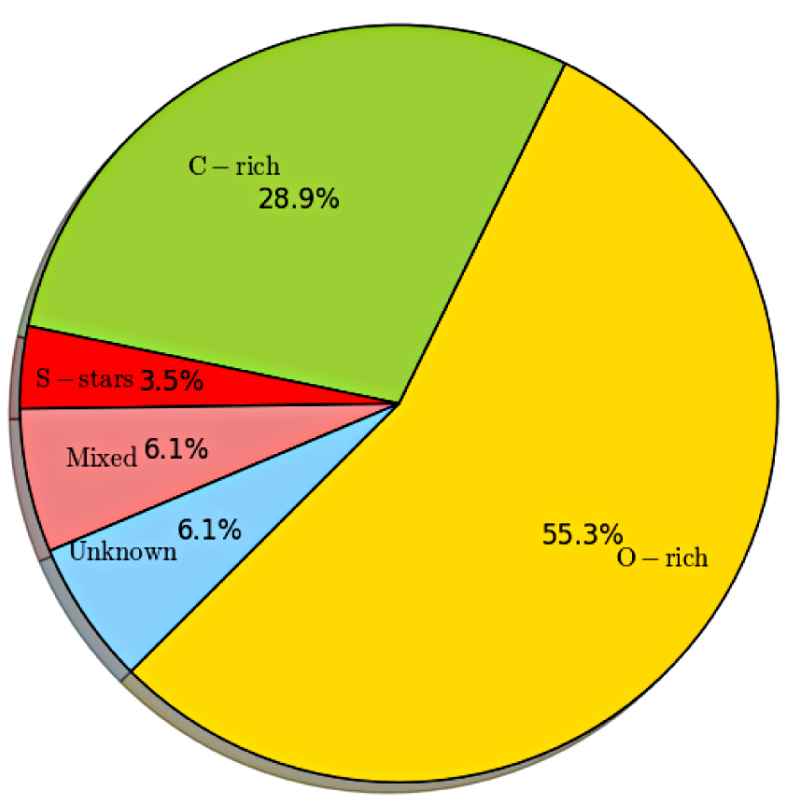

Fig. 4. Pie chart illustrating the distribution of stars in the THROES sample according to their dominant chemistry.

under this criterion while objects with $\mathrm{C} / \mathrm{O}<1$ are considered $\mathrm{O}$-rich stars. Those sources showing $\mathrm{C} / \mathrm{O}$ ratios of approximately one. are denoted as S-stars. In addition, there are also objects that present chemical indicators from both kinds of chemistries, such as the simultaneous presence of crystalline silicates, typical of O-rich chemistry, and policyclic aromatic hidrocarbons (PAHs), expected in $\mathrm{C}$-rich targets, in their mid-infrared spectra. The $\mathrm{C} / \mathrm{O}$ ratio in this kind of source may be different depending on the region of the source considered. Some of them are known to be objects in transition between O-rich and C-rich objects (Herwig 2005); others display a strong bipolar morphology, and may be surrounded by disks that could explain the mixed chemistry observed. In our sample, $29 \%$ of sources are C-rich stars, $55 \%$ are O-rich stars, $3 \%$ are S-stars, 7\% are sources with mixed chemistry, and $6 \%$ are sources with unknown chemistry (see Fig. 4).

\section{Data reduction}

The data available in the HSA have been generated using the latest version of the Standard Product Generator (SPG), an automated pipeline that takes the data from level 0 (raw) to level 2 (spectral cubes). At the time of starting this project, the archive was populated with products generated with HIPE 13.0.0.
A complete explanation of the pipeline processing steps applied in this version of the pipeline can be found in the PACS Data Reduction Guide: Spectroscopy ${ }^{5}$.

Interactive data reduction can help improve the quality of the final products by applying certain data reduction tasks not available in the automated pipeline because they require a direct interaction of the user. Therefore, we used HIPE 13.0 and version 65 of the PACS calibration files to interactively reprocess all the observations in our sample, introducing those tasks that at the moment of starting this project were not applied by the standard data reduction pipeline (version 13 at that time), with the aim of obtaining better quality products than those offered by the pipeline.

For PACS range spectroscopy observations, the interactive data reduction tasks that we have applied to improve the quality of the data can be split into two main groups:

- i) tasks applied to the spectral cubes before the level 2 data are generated, so-called, interactive re-processing;

- ii) tasks applied to the level 2 data products to extract the best 1D spectrum, so-called, post-processing.

The interactive re-processing tasks comprise:

- FlatField correction: this correction fits an n-grade polynomium to the PACS spectra to obtain a higher signal-tonoise ratio $(\mathrm{S} / \mathrm{N})$ and a better shape of the continuum.

- Telescope background correction: this correction uses the telescope background emission to flux calibrate the spectra instead of using the relative spectral response function (RSRF) applied in the SPG. This correction was later implemented in version 14 of the SPG pipeline.

To extract the final 1D spectrum from the level 2 data cubes, the following post-processing corrections were applied:

- Point source flux loss correction (PSFLC): this is needed to extract the correctly-calibrated spectrum of a point source to account for PSF losses.

- Scaling of the spectrum of the central spaxel to the continuum level of the central $3 \times 3$ superspaxel "c129": In the following we will refer to as "semi-extended $3 \times 3$ " correction as it recovers almost the whole flux of semi-extended sources, defined as those sources with a FWHM (Full Width Half Maximum) larger than a single spaxel (9.4") but small enough to fill the field of view of a single pointing (about $\left.47^{\prime \prime} \times 47^{\prime \prime}\right)$. This correction is also applied to try to minimize

\footnotetext{
5 herschel.esac.esa.int/twiki/pub/Public/
}

PacsCalibrationWeb/pacs_spec_Hipe13.pdf 
the effects of pointing jitter. The semi-extended $3 \times 3$ correction is always applied after the PSFLC and the final product is a $1 \mathrm{D}$ spectrum labelled as "PSFLC- $3 \times 3$ " corrected.

- Correction for extended sources (which we refer to as "extended $5 \times 5$ " correction): this correction was also never made available in HIPE, but we had to create it to deal with sources extended beyond the central $3 \times 3$ spaxels. It works in a similar way to the semi-extended $3 \times 3$ correction, and is also applied after PSFLC. In this case the final product is a 1D spectrum labelled as "PSFLC- $5 \times 5$ " corrected.

In addition, several objects in our sample were significantly mispointed, that is, the main target was clearly not located in the central spaxel of the $5 \times 5$ spaxel array of PACS. To extract the 1D spectra of these sources, we had to use a modified version of the semi-extended $3 \times 3$ correction, as we will explain in Sect. 3.3 in more in detail.

\subsection{Spectral flatfielding}

As explained in the PACS Data Reduction Guide, spectral flatfielding can be crucial for improving the continuum of the final spectra. For range scan observations, flatfielding will result in general in a better $\mathrm{S} / \mathrm{N}$, especially for the longer wavelength ranges. It will also remove the "fringing"-like pattern that the SPG cubes often have and it will improve the appearance of sharp rises or drops in the data.

The HIPE task used for flatfield correction is specFlatFieldRange, where the user can select the order of the polynomial to fit. The default value is five but from our experience, a lower value (three or four) usually yields better results. For PacsRange observations that do not cover the whole SED of PACS, the best results are obtained using an even lower value for the order of the polynomial (normally one). We have configured the specFlatFieldRange task to exclude the regions affected by leakage (70-73 $\mu \mathrm{m}, 98-105 \mu \mathrm{m}$, and $190-220 \mu \mathrm{m})$; by setting the option excludeLeaks to "True" we obtained "clean" spectra. The exclusion of the regions affected by leaks is actually very important to obtain a correct shape of the continuum and to improve the results obtained with the specFlatFieldRange range. We note that the improvement in the $\mathrm{S} / \mathrm{N}$ ratio after flatfielding is most remarkable in relatively faint sources.

\subsection{Telescope background correction}

This correction is needed to remove the sky and telescope background contribution. Furthermore, it uses the telescope background spectrum to flux calibrate the data, instead of using the standard calibration blocks. This process is applied using the HIPE task specDiffChop. This task computes the pairwise difference ratio: $2 *(\mathrm{On}-\mathrm{Off}) /(\mathrm{On}+\mathrm{Off})$, rather than the pairwise differences (On-Off) as is the case for the standard pipeline. This computation eliminates in an optimal way the detector drifts that appear in both On- and Off-positions. After this step, the flux density in the cubes is in units of "telescope background".

\subsection{THROES post-processing}

All the observations have been post-processed in different ways taking into account the nature of the object (extended or not) and the position of the source in the PACS FoV. The main goal of the post-processing was to extract, from the spectral cubes previously reduced, a $1 \mathrm{D}$ spectrum recovering the whole emission of the source. Depending on the post-processing tasks applied, the sources can be grouped into five main families:

- Pointed observations, good pointing, point, or semi-extended sources (PSFLC-3 $\times 3$ ): to recover the absolute flux of a point source from PACS data, it is necessary to apply first the point source flux loss correction (PSFLC) to the spectrum extracted from the central spaxel of the final (level 2) spectral cube. This correction is needed to take into account the flux losses derived from the fraction of the PSF that falls out of the central spaxel. A theoretical PSF model is used to compute the fraction of flux seen by the central spaxel and then recover the emission that has not been detected. After this, we also need to apply the semi-extended $3 \times 3$ correction in order to recover the emission received by the spaxels around the central one in case of semi-extended sources. Both corrections are performed by the HIPE task extractCentralSpectrum, which generates three different 1D spectra:

1) the first $1 \mathrm{D}$ spectrum returned by the task is simply the spectrum from the central spaxel corrected for point source flux losses;

2) the second $1 \mathrm{D}$ spectrum returned contains the integrated flux of the $3 \times 3$ central spaxels (also known as "superspaxel") with the point source flux loss correction applied to the central one;

3) finally, the third 1D spectrum is the same as the first one, corresponding to the central spaxel with the point source flux loss correction applied, scaled to the flux level of the second spectrum, that of the $3 \times 3$ superspaxel. The result is the semi-extended $3 \times 3$ corrected spectrum.

Using the Semi-extended $3 \times 3$ corrected spectrum, the whole flux from sources that are slightly extended is almost fully recovered. An example of the differences between the 1D spectrum generated after applying this correction and the spectrum taken directly from the central spaxel of the level 2 cube before post-processing is shown in Fig. 5. The vast majority of the sources in our sample have been reprocessed in this standard way and the resulting 1D spectra are displayed in Fig. A.1. An exceptional case is $\mathrm{OH} 32.8-0.3$, for which some spaxels around the central one show negative flux values and therefore these observations have only been corrected for PSFLC (i.e. the semi-extended $3 \times 3$ correction has not been applied).

- Pointed observations, extended sources (PSFLC-5 $\times 5$ ): Based on PACS photometric data, when this was available, the existing PACS spectroscopy, and the bibliography, we identified those sources in our sample that appeared more extended than just the central $3 \times 3$ spaxels. These "extended" objects are: IRAS 16122-5122, NGC 3242, NGC 40, NGC 6445, NGC 6543, NGC 6781, NGC 6826, NGC 7009, NGC 7026, and Mz3. Due to their extension, their emission can spread sometimes over the whole PACS FoV and, therefore, for these objects, we developed a new task, the extended $5 \times 5$ correction, to scale the spectrum from the central spaxel, after applying the point source flux loss correction, to the continuum level of the $55 \times 5$ spectrum, as we did for the semi-extended $3 \times 3$ correction.

As we will see in Sect. 5, with this new task we were able to obtain a more realistic spectrum of the extended sources in our sample, better than the spectrum generated with the semi-extended $3 \times 3$ correction, as in. this way we recovered the whole emission from the source. In Fig. A.2, we show the final 1D spectra of these extended sources after applying the 

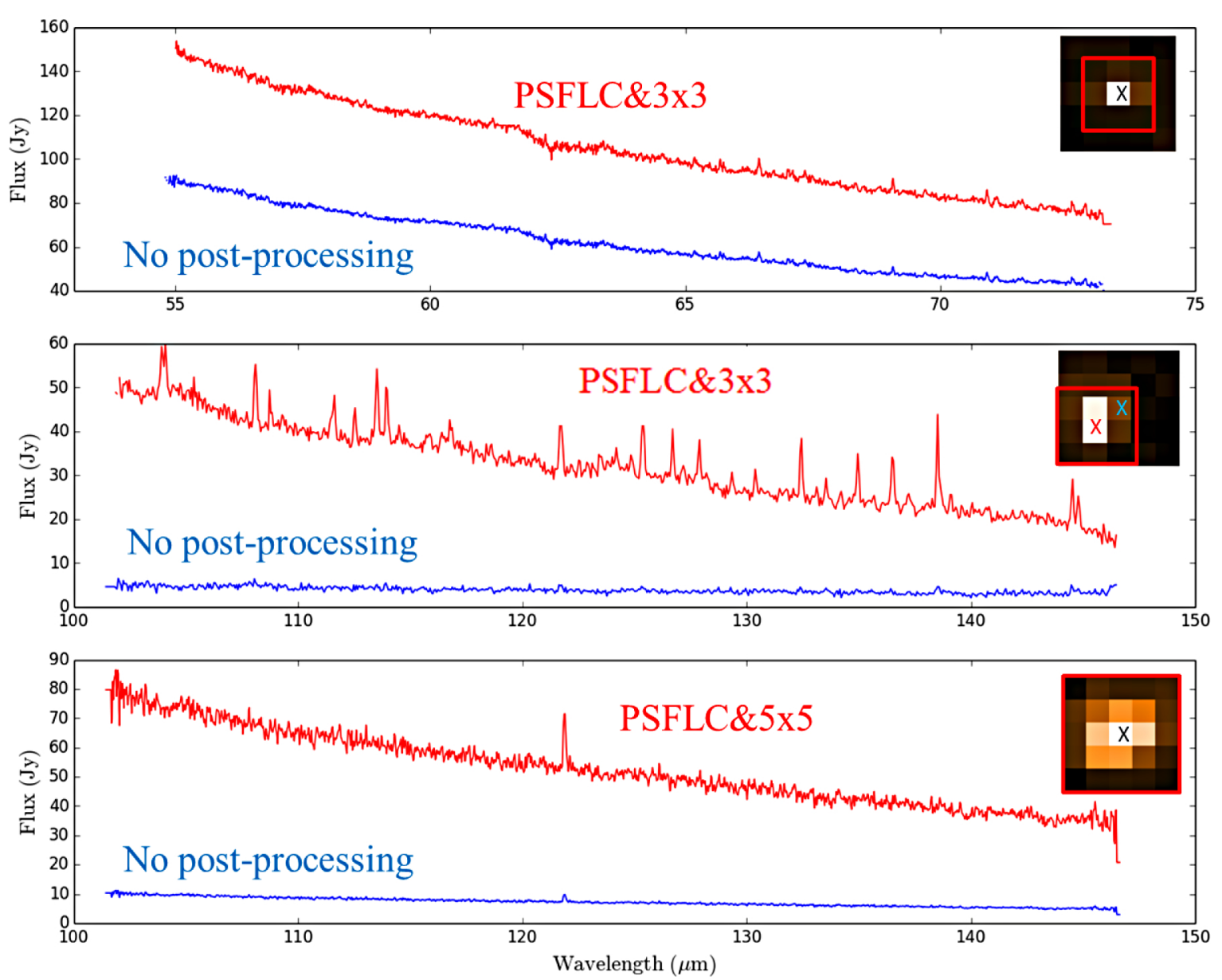

Fig. 5. One-dimensional spectra extracted for three different targets in the THROES catalogue following the procedures described in Sect. 3 (red). The flux corrections and apertures have been chosen depending on the source extent and its location in the $5 \times 5$ spaxels FoV of PACS. For comparison, we show the corresponding spectrum obtained in each case from the central spaxel before post-processing (blue). At the top right corner of each box, we show a layer of the FinalCube with the $5 \times 5$ spaxels of the PACS spectrometer of the observation. The crosses indicate the spaxel from which the spectra were taken; a black cross means that both spectra were extracted from the central spaxel. The squares (red and blue) enclose the spaxels used for the $3 \times 3$ correction or for the $5 \times 5$ correction. Top: AFGL 3116, blue camera of PACS (ObsID: 1342212512): well pointed and semi-extended source; PSFLC- $3 \times 3$ correction has been applied. Center: same as above but for IRC-10529, red camera of PACS (ObsID: 1342208931): this is a mispointed observation where the blue spectrum corresponds to that extracted from the central spaxel without post-processing, while the red one is the spectrum taken from the brightest spaxel and with the post-processing applied (PSFLC-3 $\times 3$ ). Bottom: same as the first two panels but for NGC 6543, red camera (ObsID: 1342238389): this is an extended source; PSFLC- $5 \times 5$ correction applied.

PSFLC and the extended $5 \times 5$ correction. The effect of this task in terms of continuum flux level recovery can be seen in Fig. 5.

- Pointed observations, mispointing (PSFLC-3 $\times 3$ ): in our sample there were also seven cases of mispointed observations, namely, IRAS17347-3139, NGC 6302, IRC-10529, OH21.5+0.5, AFGL 5379, IRAS 16279-4757, and IRAS 13428-6232, for which the source is located on a spaxel different than the central one. In these cases, extractCentralSpectrum cannot be applied because, in this task, the $3 \times 3$ superspaxel is always built around the central spaxel, instead of around the one where the source is located. To deal with these mispointed cases, we developed a script that works in a similar way to the HIPE task extractCentralSpectrum but which allows the user to select the spaxel (other than the central one) where the source is located. Our task was successfully applied to IRC-10529, OH 21.5+0.5, IRAS 13428-6232, NGC 6302, and IRAS 17347-3139. In Fig. A. 3 we show the final 1D spectra obtained after applying this correction.
For AFGL 5379 and IRAS16279-4757 this correction did not work correctly as, due to the position of the sources being too far away from the central spaxel, it was impossible to generate the $3 \times 3$ superspaxel around the off-centred spaxel where the source was located.

As was done for other observations in our sample, it is also necessary to correct these mispointed observations from PSF losses before applying the semi-extended $3 \times 3$ correction described above. To do that, HIPE provides two different tasks: extractSpaxelSpectrum and pointSourceLossCorrection. The first one takes the spectrum from the spaxel where the source is located and, after that, the second one corrects for the point source flux losses.

Again in Fig. 5 we show the significant improvement of the final 1D spectra after applying the semi-extended $3 \times 3$ correction to mispointed observations.

- Sources corrected only for PSFLC: three additional sources(AFGL 5379, IRAS 16279-4757, and OH 32.8-0.3) were only corrected for PSFLC, due to different issues that prevented the application of the semi-extended 


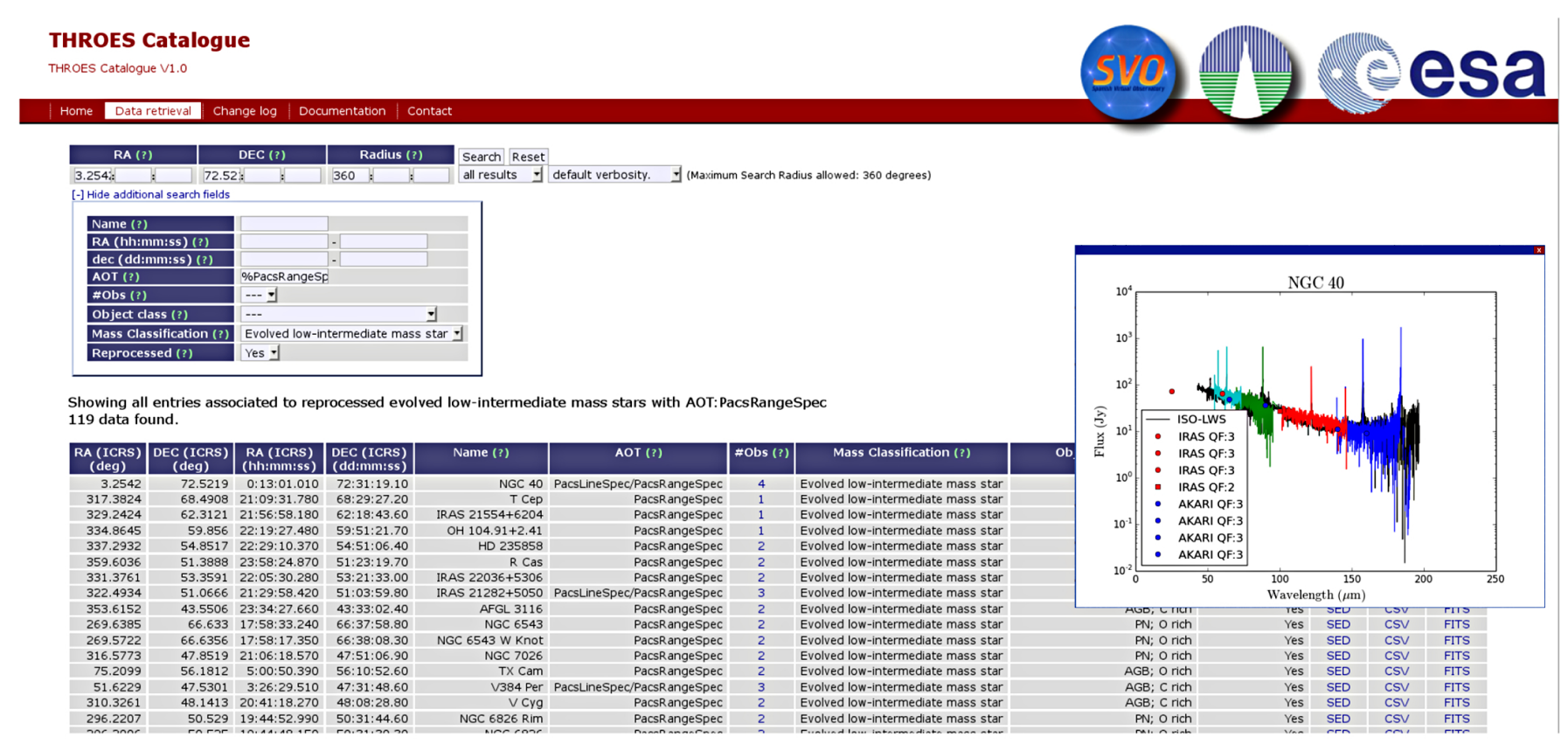

Fig. 6. Screenshot of the THROES catalogue web interface. All the columns and search fields described in Sect. 4 are shown as well as an example of a complete SED with IRAS and AKARI data overplotted.

\begin{abstract}
$3 \times 3$ correction. These issues are related to the position of the source in the $5 \times 5$ spaxels array and the presence of corrupted data in some of the spaxels needed to create the $3 \times 3$ superspaxel. Their spectra are displayed in Fig. A.4. For these three sources the absolute flux level of the final 1D spectrum available in the THROES catalogue should be considered only as a lower limit.
\end{abstract}

To summarize, after our interactive data reduction process, we generated for each object the following final products:

- 1) Final spectral cubes (level 2) with the improvements that result from flatfielding and telescope background correction.

- 2) 1D spectra with the PSFLC applied, as well as the specific correction for slightly extended sources (semi-extended $3 \times 3$ correction) or extended sources (extended $5 \times 5$ correction). As a word of caution, we note that the scaling strategy followed assumes that the extension of the continuum and of the line-emitting region is roughly the same and that the spectral lines emission does not vary within the nebula, which may not be the case in some of the more extended objects. For those sources showing a more complex and extended morphology (particularly the PSFLC $-5 \times 5$ corrected targets), we recommend the users to create their own 1D spectra making use of the final cubes provided in the THROES catalogue if they want to perform a more detailed analysis.

\section{THROES catalogue}

All the reprocessed PACS range spectroscopy observations have been compiled in a catalogue that is accessible via a web interface available at https://throes.cab.inta-csic.es/. On this web page all the reprocessed data are publicly available, including some general information about the objects and the observations made, as well as plots with the reduced PACS spectroscopy data and complementary spectroscopic and photometric observations from other observatories. A screenshot of the "Data Retrieval" interface of the catalogue is shown in Fig. 6.
The tool used to create the web-based catalogue is SVOCat ${ }^{6}$. This application, developed by the Spanish Virtual Observatory $\left(\mathrm{SVO}^{7}\right)$ is designed to make the publication of an astronomical catalogue easier, both as a web page and as a Virtual Observatory Cone Search service. Our archive system has been designed following the IVOA (International Virtual Observatory Alliance $)^{8}$ standards and requirements. In particular, it implements the Cone Search protocol, a standard defined by the IVOA for retrieving records from a catalogue of astronomical sources. Queries made through the Cone Search service are based on the description of a sky position and an angular distance, defining a cone on the sky. The response returns a list of astronomical sources from the catalogue whose positions in the sky lie within the pre-defined cone, formatted as a VOTable (Virtual Observatory Table). The system as a whole is included in the IVOA Registry and can therefore be discovered by any VO-tool.

To structure our catalogue in a homogeneous and logical way, all the observations with the same pointing have been grouped into a unique entry in the archive. Therefore, there is a single line per region in the sky observed. As we can see in Fig. 6, there are 13 columns for each entry, each providing information about the object or the observation as well as links to plots and to the reprocessed data. Each of the columns contains the following information:

- Columns 1 to 4: the equatorial coordinates (right ascension and declination) of the observation in different units: decimal degrees in Cols. 1 and 2, and hh:mm:ss and dd:mm:ss in Cols. 3 and 4.

- Column 5: name of the object in the THROES catalogue.

- Column 6: the astronomical observation template (AOT). This could be PacsRange or PacsLine. For some objects there are spectra taken in both modes. These cases appear in the catalogue as PacsLine/PacsRange. Only the PacsRange

\footnotetext{
6 http://svo2.cab.inta-csic.es/vocats/Svocat-doc/

http://svo.cab.inta-csic.es

8 http://www.ivoa.net
} 
spectra have been reprocessed in the current version of the THROES catalogue.

- Column 7: the number of observations taken in that position of the sky. By clicking on the number shown in this column, a new table is deployed with detailed information for each observation, such as: target name, equatorial coordinates (RA and Dec), proposal name, AOT, observation ID, observing date and time, and original AOR (Astronomical Observation Request) label.

- Column 8: mass classification (based on the bibliography and $\mathrm{SIMBAD}^{9}$ ). The options are: evolved low-intermediate mass star, evolved massive star, or unknown.

- Column 9: object classification including the evolutionary stage and its dominant chemistry (based on the bibliography and SIMBAD), the options are: O-rich AGBs, C-rich AGBs, $\mathrm{S}$ stars, OH/IR stars, O-rich post-AGBs, C-rich post-AGBs, mixed chemistry post-AGBs, O-rich $\mathrm{PNe}, \mathrm{C}$-rich $\mathrm{PNe}$, mixed chemistry PNe, or Unknown.

- Column 10: this column indicates if the observations have been reprocessed under the THROES project or not. This is because THROES may be expanded in the future to other observing modes of PACS and/or massive evolved stars.

- Column 11: by double clicking on "SED", a pop-up window is displayed showing the 1D PACS spectra generated after the interactive data reduction and subsequent post-processing, together with complementary photometric (IRAS and AKARI) and spectroscopic (Infrared Space Observatory-Long-Wave Spectrometer (ISO-LWS)) data, when available.

- Column 12: by double clicking on "CSV" (CommaSeparated Values), a compressed folder with the name of the target is downloaded containing a gzipped tar file with the 1D PACS spectra, in CSV format.

- Column 13: similarly, by double clicking on "FITS" (Flexible Image Transport System), a compressed gzipped tar file is downloaded containing the THROES reprocessed final spectral cubes (level 2) in FITS format and the 1D PACS spectra, also in FITS format, derived from these final spectral cubes after post-processing.

More information about how to query the catalogue through the different search fields is available in the documentation available on the THROES catalogue web page.

\section{Characterization of the THROES sample}

All the individual THROES spectra are shown from Figs. A.1 to A.4. To illustrate the quality of the resulting spectra, every plot contains not only the final PACS 1D spectra, but also the photometric (IRAS and AKARI) and spectroscopic ISO-LWS data, whenever these are available. In Table A.1 we display information associated to the PACS, ISO (Infrared Space Observatory), IRAS, and AKARI data used for the generation of the SEDs.

\subsection{Comparison with IRAS and AKARI photometry}

All the objects in the THROES catalogue have complementary IRAS photometric information while, for AKARI data, the percentage of common objects decrease to $89 \%$. The Infrared Astronomical Satellite (IRAS) ${ }^{10}$ point source catalogue presents photometric data in four bands centred at 12, 25, 60, and $100 \mu \mathrm{m}$.

\footnotetext{
9 http://simbad.u-strasbg.fr/simbad/

10 http://irsa.ipac. caltech. edu/IRASdocs/iras.html
}

The beam size of IRAS observations, which is much larger than that of PACS, varies from $2^{\prime}$ at shorter wavelengths to $5^{\prime}$ at longer ones (Jeong et al. 2007). AKARI ${ }^{11}$ covers longer wavelengths, $65,90,140$, and $160 \mu \mathrm{m}$, and the beam size varies from $0.5^{\prime}$ to $0.9^{\prime}$ (Jeong et al. 2007). The different beam sizes of the instruments is a key point that has to be kept in mind when comparing PACS spectroscopy to IRAS and AKARI photometric data.

In order to check the quality of the final processed PACS spectroscopy data and to obtain information about the effect of the post-processing tasks applied to generate the final 1D spectra, a comparison between the synthetic photometry derived from the PACS spectroscopic data and the IRAS and AKARI photometric data at 100 and $160 \mu \mathrm{m}$, respectively, has been done.

Synthetic photometry is generated by convolving the transmission curve of the photometric filters, IRAS ${ }_{100}$ and AKARI $_{160}$, with the 1D PACS spectra ${ }^{12}$.

As the synthetic photometry requires a convolution between the 1D PACS spectrum and the curve of the photometric filters, it is necessary that the PACS spectrum spectral coverage extends, at least, along the whole wavelength subrange covered by the photometric filters. For this reason, the synthetic photometry can be estimated for the subsample of 71 THROES targets with full spectral coverage of the PACS wavelength range.

As mentioned in Sect. 3, due to the instrument configuration and the exclusion of some sub-regions affected by leakage, all the 1D PACS spectra show a small gap (less than $10 \mu \mathrm{m}$ ) around $100 \mu \mathrm{m}$, even for those sources that present a complete spectral coverage. As the photometric curve of IRAS $100 \mu \mathrm{m}$ is centred on this region, it is important to find a solution. To cope with that in the synthetic photometry estimation, a linear interpolation was done to approximate the continuum flux level in this region. This assumption is good enough as intense emission lines are not expected in this region.

On the AKARI $160 \mu \mathrm{m}$ side, due to the exclusion of the wavelength regions affected by leakage, there are no PACS data to cover the wavelength range from 190 to $220 \mu \mathrm{m}$. To solve that, a power law $\left(\lambda^{\alpha}\right)$ was fitted to the red bands of the PACS SED and, after that, we extrapolated the flux values to the 190-220 $\mu \mathrm{m}$ region. At these wavelengths, we are mainly tracing the Rayleigh-Jeans emission, so this extrapolation is reasonable.

To show the effect of the post-processing tasks applied in the THROES reduction process, the synthetic photometry has been estimated for 1D PACS spectra before and after applying the post-processing tasks. In Fig. 7 the comparison between IRAS ${ }_{100}$ and $\mathrm{AKARI}_{160}$ photometric data and their synthetic counterparts using PACS data with and without post-processing is shown. We can see clearly that a disagreement between the photometric and the synthetic photometric data, before post-processing, is obtained. This disagreement is significantly reduced after postprocessing tasks are applied, so it confirms that the tasks introduced in the reduction process of THROES are needed for reliable absolute flux calibration. The few points that fall out of a one-to-one observational-to-synthetic photometry relation are points that have bad IRAS and/or AKARI photometric data, (Quality Flag $\neq 3$, red triangles) or are associated to extended objects (blue crosses).

\footnotetext{
${ }^{11}$ http://www.ir.isas.jaxa.jp/AKARI/index.html

12 The transmission curve of IRAS $_{100}$ has been obtained from: http://irsa.ipac. caltech.edu/IRASdocs/exp.sup/ch2/ tabC5.html and the transmission curve of AKARI 160 has been estimated from the relative response function available in: http: //svo2. cab. inta-csic.es/theory/fps3/?id=AKARI/FIS. N160
} 

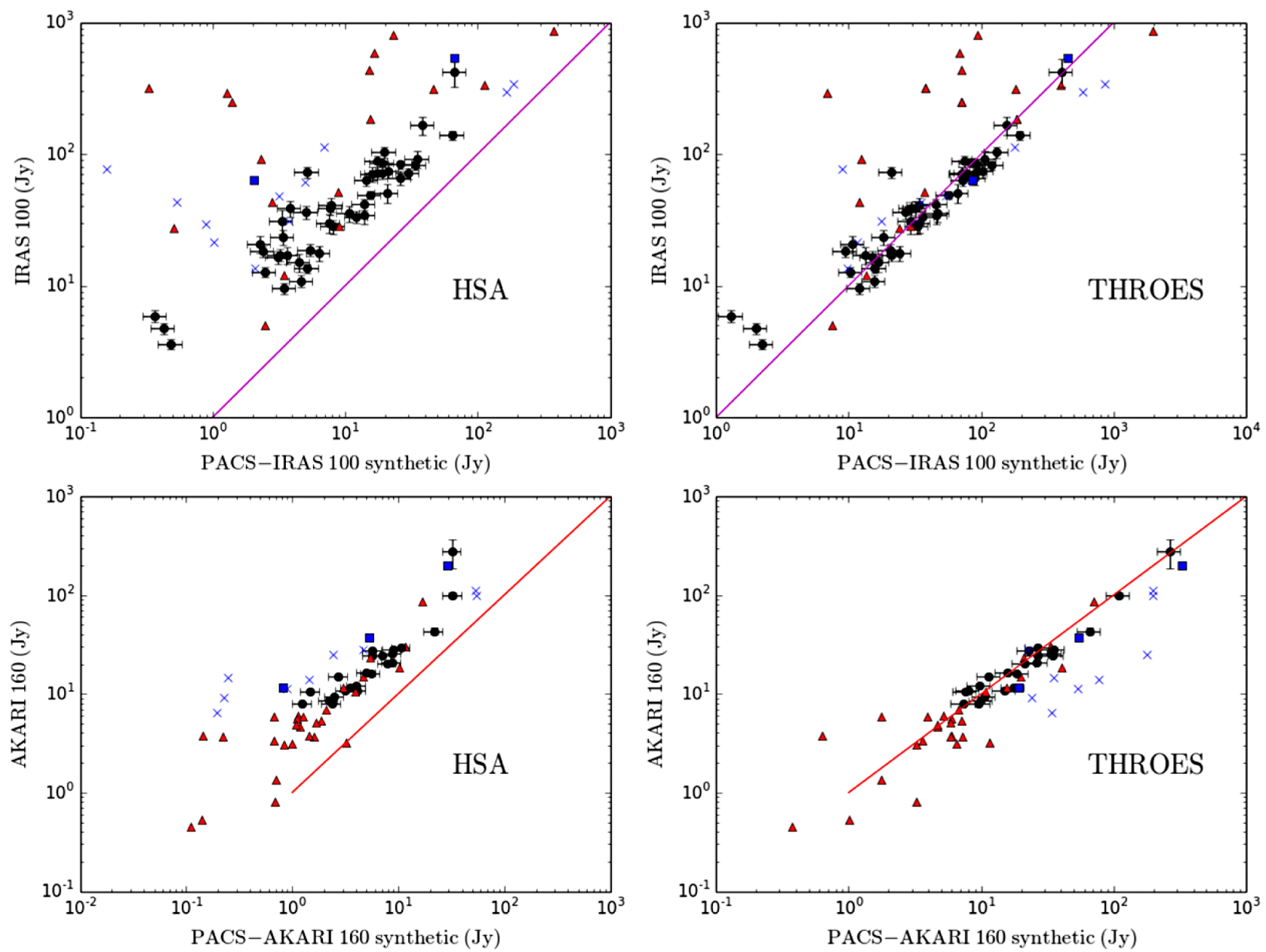

Fig. 7. Comparison of IRAS $(100 \mu \mathrm{m})$ and AKARI $(160 \mu \mathrm{m})$ photometry with synthetic PACS photometry at these wavelengths before (left) and after (right) the interactive reprocessing of the data carried out in this work and that is available through the THROES catalogue. The objects plotted here are those which present a complete coverage of the PACS spectral range. We have established a code to distinguish the extended objects (blue crosses), the mispointed ones (red squares), the objects with bad IRAS ${ }_{100}$ or AKARI 160 data (Quality Flag $\neq 3$, red triangles), and objects with good IRAS ${ }_{100}$ or AKARI ${ }_{160}$ data (Quality Flag = 3, black circles with error bars). The solid line represents a perfect match (1:1 ratio) of the synthetic (PACS) and observational photometric (IRAS and AKARI) points.

\subsection{ISO-LWS spectroscopy}

It is interesting to compare our PACS spectroscopic data to ISOLWS spectra, since this instrument covers a wavelength range from 43 to $197 \mu \mathrm{m}$ that almost fully overlaps with PACS. However, it is important to keep in mind that ISO-LWS presents a worse spectral resolution, $R=200$ (medium resolution) and $R=1000$ (high resolution), and a larger beam size $\left(80^{\prime \prime} \times 100^{\prime \prime}\right)$.

Whenever available, ISO spectra are shown in Figs. A.1 to A.4 together with our 1D PACS spectra and IRAS/AKARI photometry points. After a visual inspection of those objects which present both PACS and ISO data, objects can be grouped into five main families:

1. Good agreement between PACS and ISO: for most of the sources, a good agreement between PACS and ISO-LWS spectroscopic data is found. For example, HD 161796, CIT 6, AFGL 618, or CPD-568032 (see Fig. A.1).

2. Mispointed PACS observations without $3 \times 3$ correction: due to its location close to the edge of the PACS $5 \times 5$ array, it was not possible to apply the semi-extended $3 \times 3$ correction to one source, AFGL 5379. This could explain why the continuum flux level of the ISO-LWS is higher than that of the PACS spectrum (see Fig. A.4).
3. Background contamination of the ISO spectra: one of the most remarkable facts found with this comparison is that, for some sources, the ISO-LWS spectra show a continuum level higher than the one of PACS. This effect seems to be more evident at longer wavelengths $(>100 \mu \mathrm{m})$. Besides, for all these spectra we find an emission line at $158 \mu \mathrm{m}$ associated to [C II] due to interstellar emission, so the origin of this extra contribution could be the contamination of the interstellar medium. There are 11 sources that have been classified in this group: AFGL6815, IRAS 16342-3814, IRAS 16594-4656, IRAS 22036+5306, IRAS 21282+5050, MWC 922, NGC 6537, NML Cyg, OH 26.5+0.6, OH 32.80.3 , and V Cyg. The reason why ISO-LWS spectra show this contamination is the larger FoV of ISO-LWS with respect to the PACS FoV (see Figs. A.1 to A.4).

4. Very extended sources: extended sources in THROES sample have been corrected using the extended $55 \times 5$ correction to recover the whole flux taken by PACS spectroscopy. However, there are some objects, such as NGC 6781, that are even larger than the PACS FoV and, therefore, PACS spectroscopic data do not measure all the flux emission from the source in contrast to ISO-LWS, which has a larger beam. For that reason, for these very extended sources, the continuum 
flux level of the ISO-LWS spectra is higher than that of PACS (see Fig. A.2).

5. Bad ISO data: finally, for some sources like IRAS $07027-$ 7934 or HD 56126, ISO-LWS data present evident artefacts that make the ISO spectroscopic data unreliable (see Fig. A.1).

\section{Summary}

We made an inventory of all the observations of all evolved stars taken in standard mode by Herschel with PACS spectroscopy. From all of them, we selected pointed, Chop/Nod, and PacsRange observations of evolved low-to-intermediate mass stars and we interactively processed the resulting 220 individuals Herschel/PACS Obs IDs, corresponding to 114 different targets.

Along the reduction process we introduced new tasks to improve the final PACS spectroscopy products. These tasks can be divided into two main groups:

- 1) Tasks applied to the spectral cubes before the level 2 data are generated: flatfield correction and telescope background correction.

- 2) Tasks applied to the level 2 data products to extract the best $1 \mathrm{D}$ spectrum (post-processing). These tasks try to recover the whole flux of the sources within the PACS $5 \times$ 5 spaxels array, taking into account the extension of the emission and the position of the object in the PACS FoV. They are: point source flux loss correction (PSFLC), semiextended $3 \times 3$ correction, and extended $55 \times 5$ correction.

After the interactive data reduction, we generated synthetic photometry to compare our final THROES 1D spectra to photometric, IRAS 100 , and AKARI 160 photometric data. THROES 1D spectra were compared also with spectroscopic (ISO-LWS) data. From these comparisons we can conclude that our re-processing generates improved quality products compared with those routinely generated by the SPG in an automated mode, available in the HSA, and are in good agreement with IRAS and AKARI photometric data. Furthermore, the comparison of PACS with ISO-LWS spectra has highlighted the presence of interstellar contamination in some ISO-LWS data.

To ease the access to the final spectral cubes (level 2) and to the post-processed 1D spectra, we have created a webbased interface using SVOCat. Through https://throes. cab.inta-csic.es/, all the products generated as a result of the THROES interactive data reduction process are available. The THROES catalogue is expected to be updated in the near future by extending the analysis to new spectroscopic data from other instruments such as SPIRE. Furthermore, there is the potential to extend the analysis to evolved massive stars.

Acknowledgements. The Herschel spacecraft was designed, built, tested, and launched under a contract to ESA managed by the Herschel/Planck Project team by an industrial consortium under the overall responsibility of the prime contractor Thales Alenia Space (Cannes), and including Astrium (Friedrichshafen) responsible for the payload module and for system testing at spacecraft level, Thales Alenia Space (Turin) responsible for the service module, and Astrium (Toulouse) responsible for the telescope, with in excess of a hundred subcontractors. HCSS and HIPE are joint developments by the Herschel Science Ground Segment Consortium, consisting of ESA, the NASA Herschel Science Center, and the HIFI, PACS, and SPIRE consortia. We are grateful to the entire spectroscopy group of PACS for their help and support, especially to E. Puga and $\mathrm{K}$. Exter, as well as the anonymous referee for their careful reading of the manuscript and very useful suggestions. This research has been supported by the funding of the ESAC Faculty and the Herschel Science Division. C.S.C. is partially funded by the Spanish MINECO through grants AYA2012-32032 and AYA2016-750066-C2-1-P. This research has made use of the Spanish Virtual Observatory (svo.cab.inta-csic.es) supported from the Spanish MINECO through grants AyA2014-55216.

\section{References}

Aleman, I., Ueta, T., Ladjal, D., et al. 2014, A\&A, 566, A79

Balick, B., \& Frank, A. 2002, ARA\&A, 40, 439

Danilovich, T., Teyssier, D., Justtanont, K., et al. 2015, A\&A, 581, A60

De Beck, E., Decin, L., de Koter, A., et al. 2010, A\&A, 523, A18

de Graauw, T., Helmich, F. P., Phillips, T. G., et al. 2010, A\&A, 518, L6

de Vries, B. L., Klotz, D., Lombaert, R., et al. 2011, in Why Galaxies Care about AGB Stars II: Shining Examples and Common Inhabitants, eds. F. Kerschbaum, T. Lebzelter, \& R. F. Wing, ASP Conf. Ser., 445, 621

Decin, L., Justtanont, K., De Beck, E., et al. 2010, A\&A, 521, L4

García-Hernández, D. A., Perea-Calderón, J. V., Bobrowsky, M., \& GarcíaLario, P. 2007, ApJ, 666, L33

Griffin, M. J., Abergel, A., Abreu, A., et al. 2010, A\&A, 518, L3

Groenewegen, M. A. T., Waelkens, C., Barlow, M. J., et al. 2011, in Why Galaxies Care about AGB Stars II: Shining Examples and Common Inhabitants, eds. F. Kerschbaum, T. Lebzelter, \& R. F. Wing, ASP Conf. Ser., 445,567

Habing, H. J. 1996, A\&ARv, 7, 97

Herwig, F. 2005, ARA\&A, 43, 435

Jeong, W.-S., Nakagawa, T., Yamamura, I., et al. 2007, PASJ, 59, S429

Kwok, S. 2005, J. Korean Astron. Soc., 38, 271

Maercker, M., Danilovich, T., Olofsson, H., et al. 2016, A\&A, 591, A44 Pilbratt, G. L., Riedinger, J. R., Passvogel, T., et al. 2010, A\&A, 518, L1

Poglitsch, A., Waelkens, C., Geis, N., et al. 2010, A\&A, 518, L2

Ueta, T., Ladjal, D., Exter, K. M., et al. 2014, A\&A, 565, A36

van der Veen, W. E. C. J., \& Habing, H. J. 1988, A\&A, 194, 125 


\section{Appendix A}
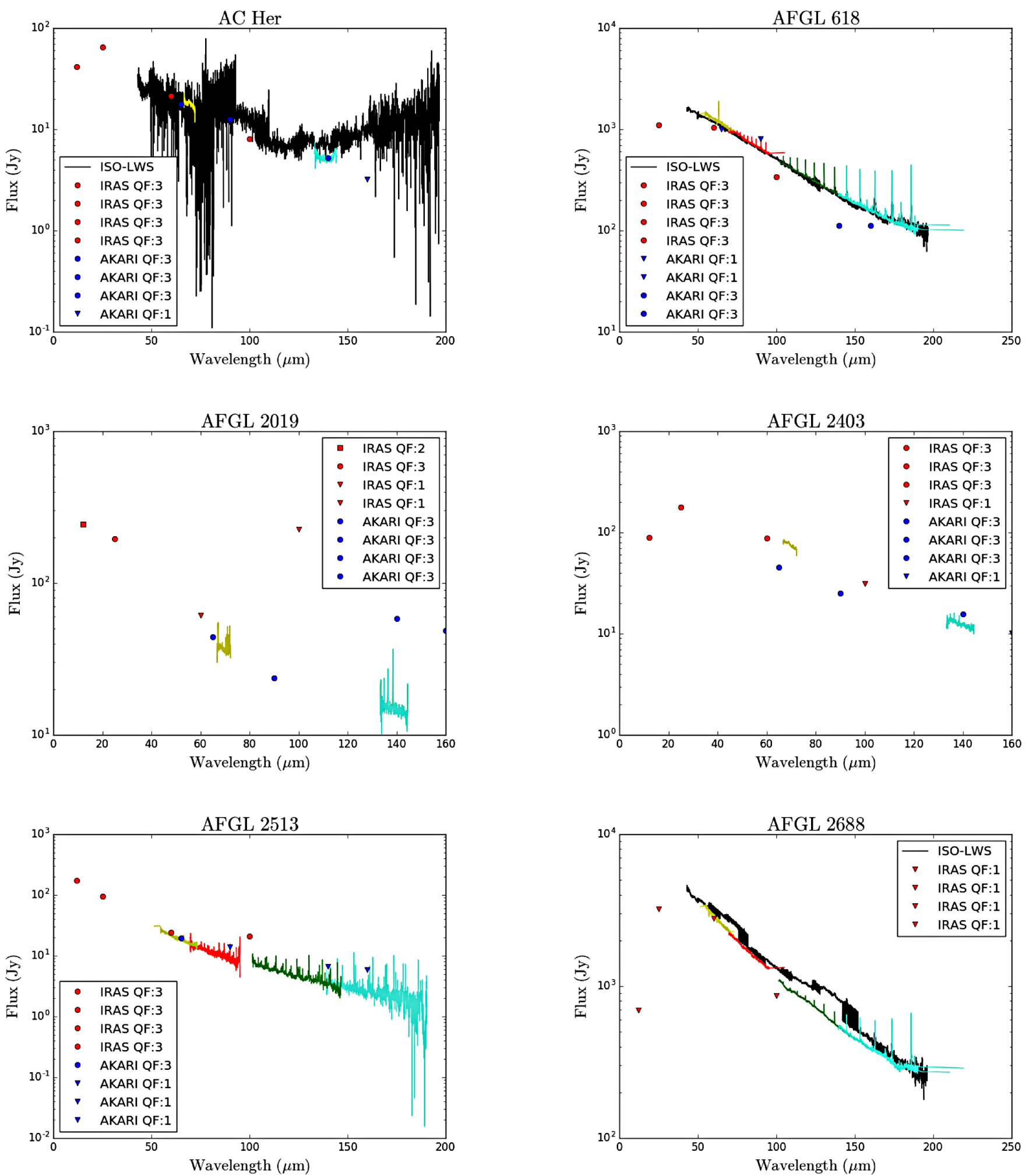

Fig. A.1. PACS spectroscopy SED of well-pointed, non-extended sources, after applying PSFLC and semi-extended $3 \times 3$ correction (PSFLC$3 \times 3$ ). PACS data is colour-coded according to the spectral region covered by each subrange, as follows: $\sim 50-70 \mu \mathrm{m}$ (yellow), 70-100 $\mu \mathrm{m}$ (red), $\sim 100-145 \mu \mathrm{m}$ (green), and $\sim 145-200 \mu \mathrm{m}$ (turquoise). IRAS (red points) and AKARI (blue points) photometric data and ISO (black) spectroscopic data are also displayed when available. We do not show those observations pointed to a specific region in the case of very extended sources such as rims or knots. 

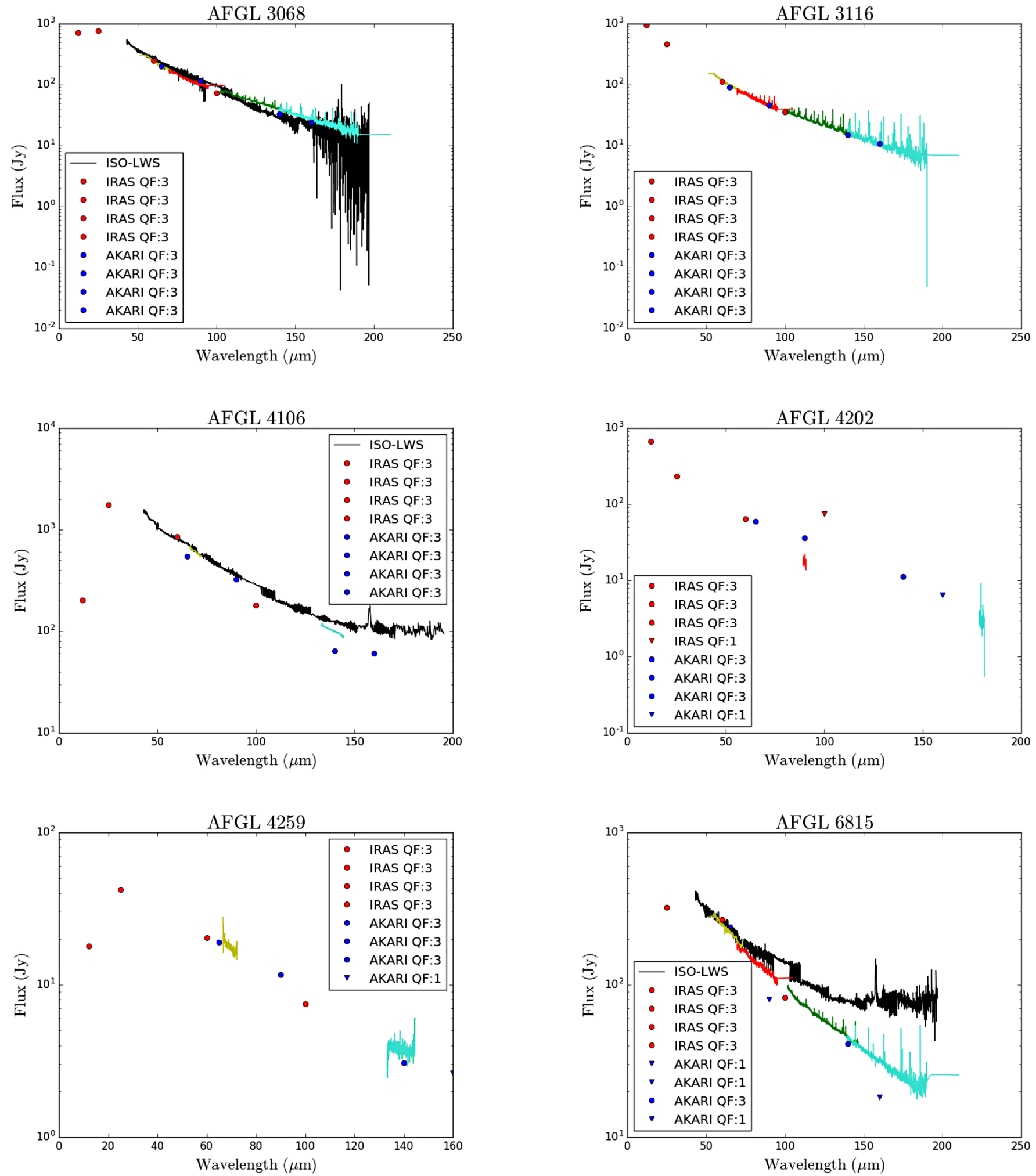

Fig. A.1. continued. 

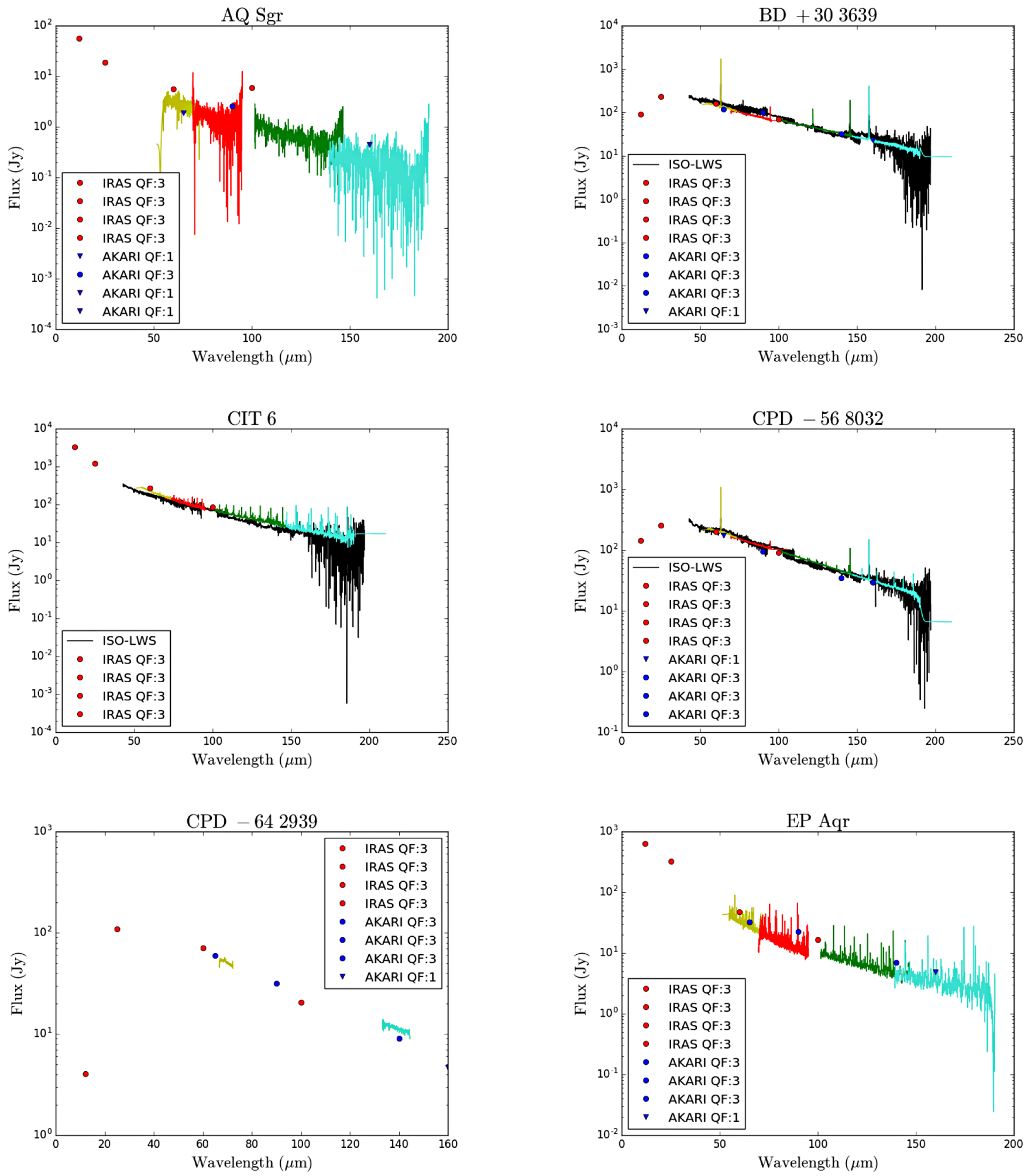

Fig. A.1. continued. 

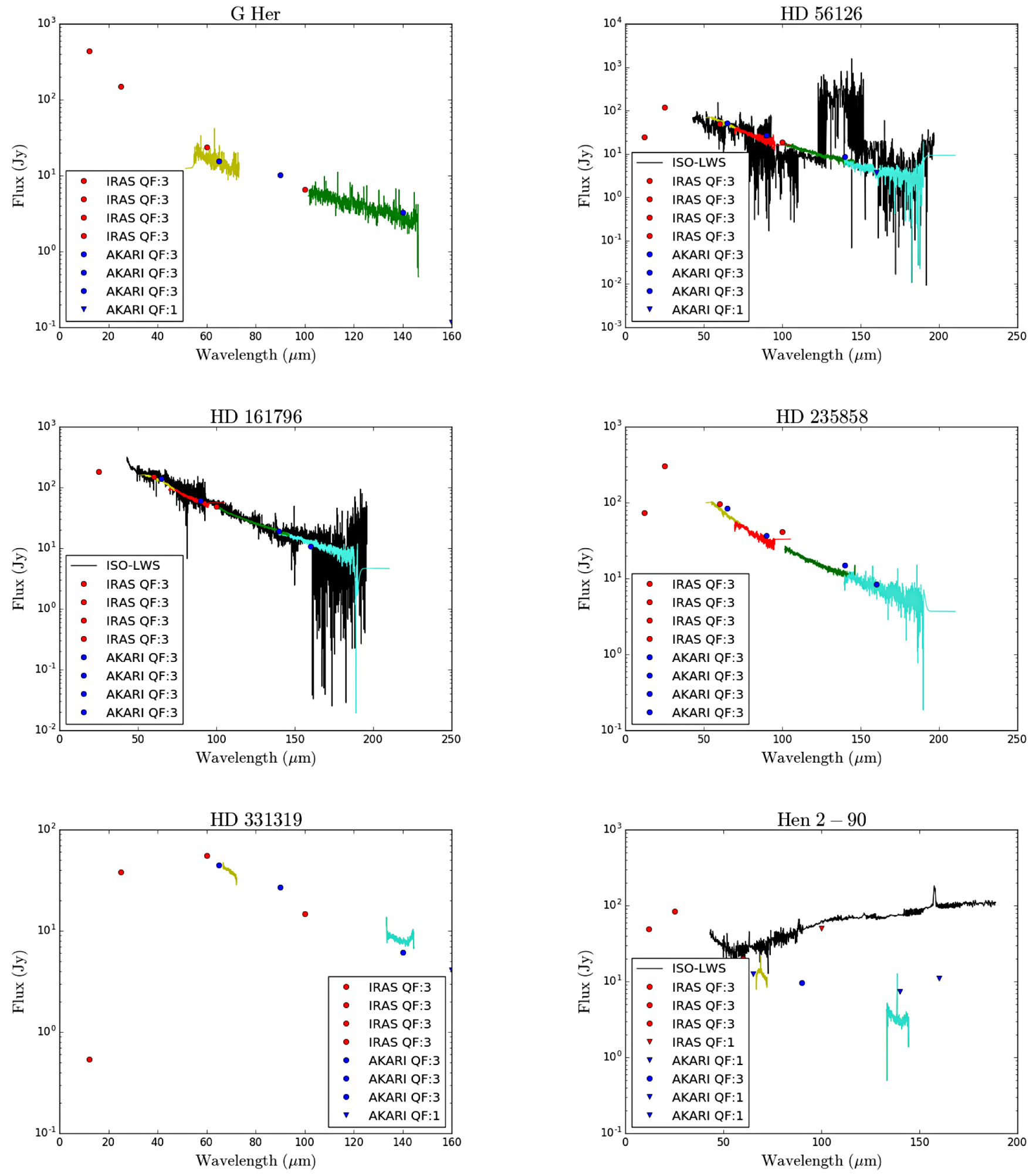

Fig. A.1. continued. 

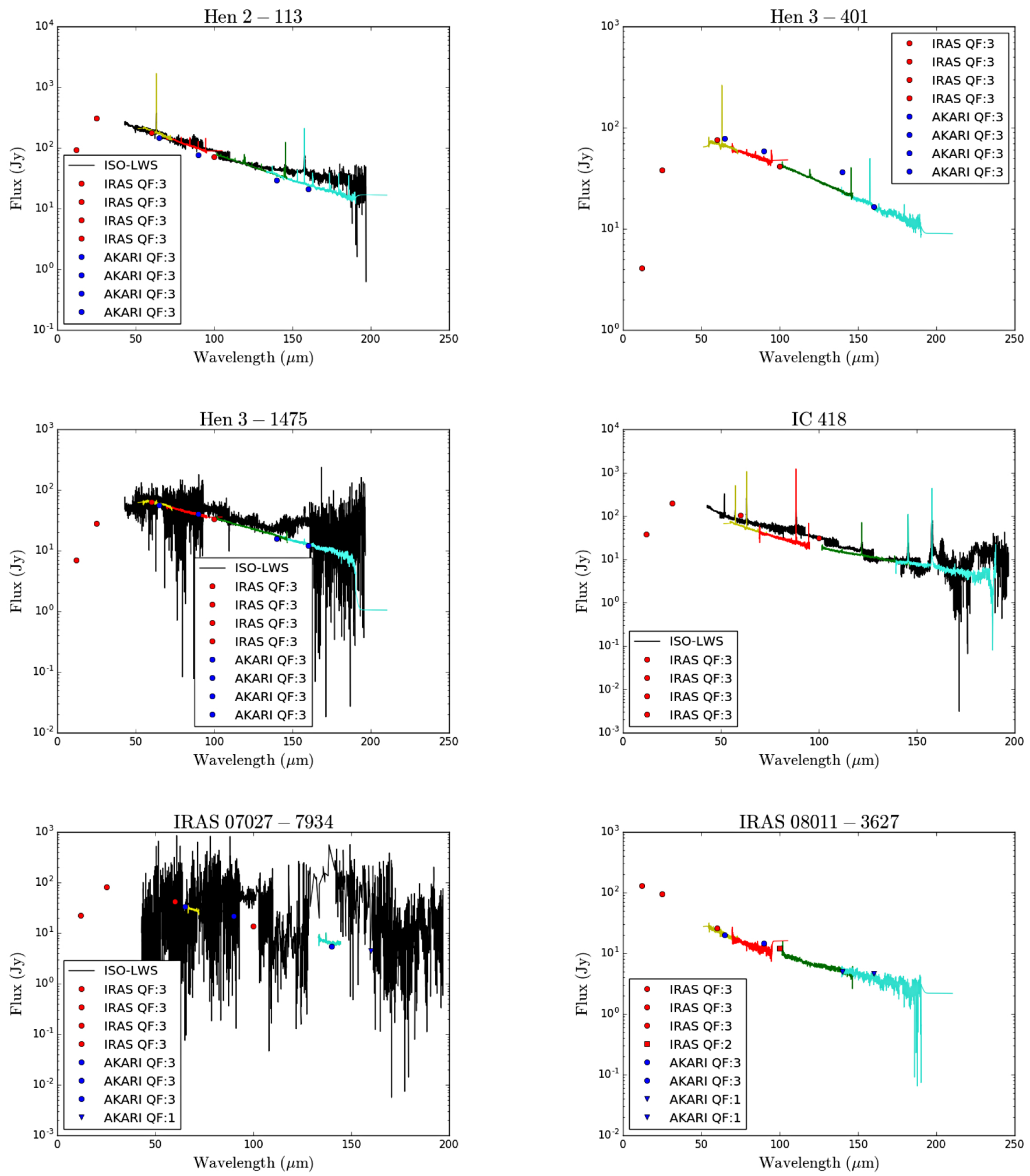

Fig. A.1. continued. 

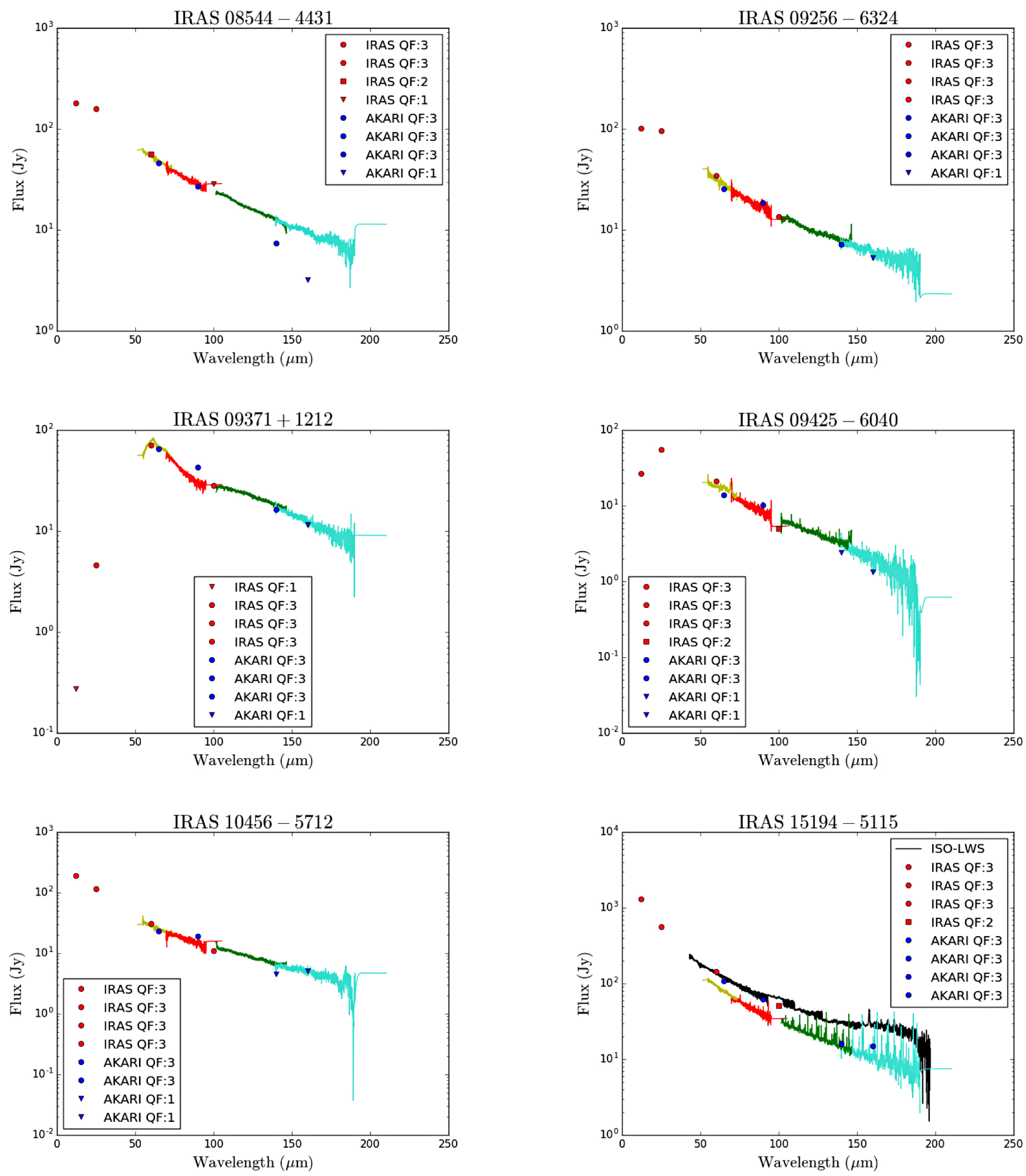

Fig. A.1. continued. 

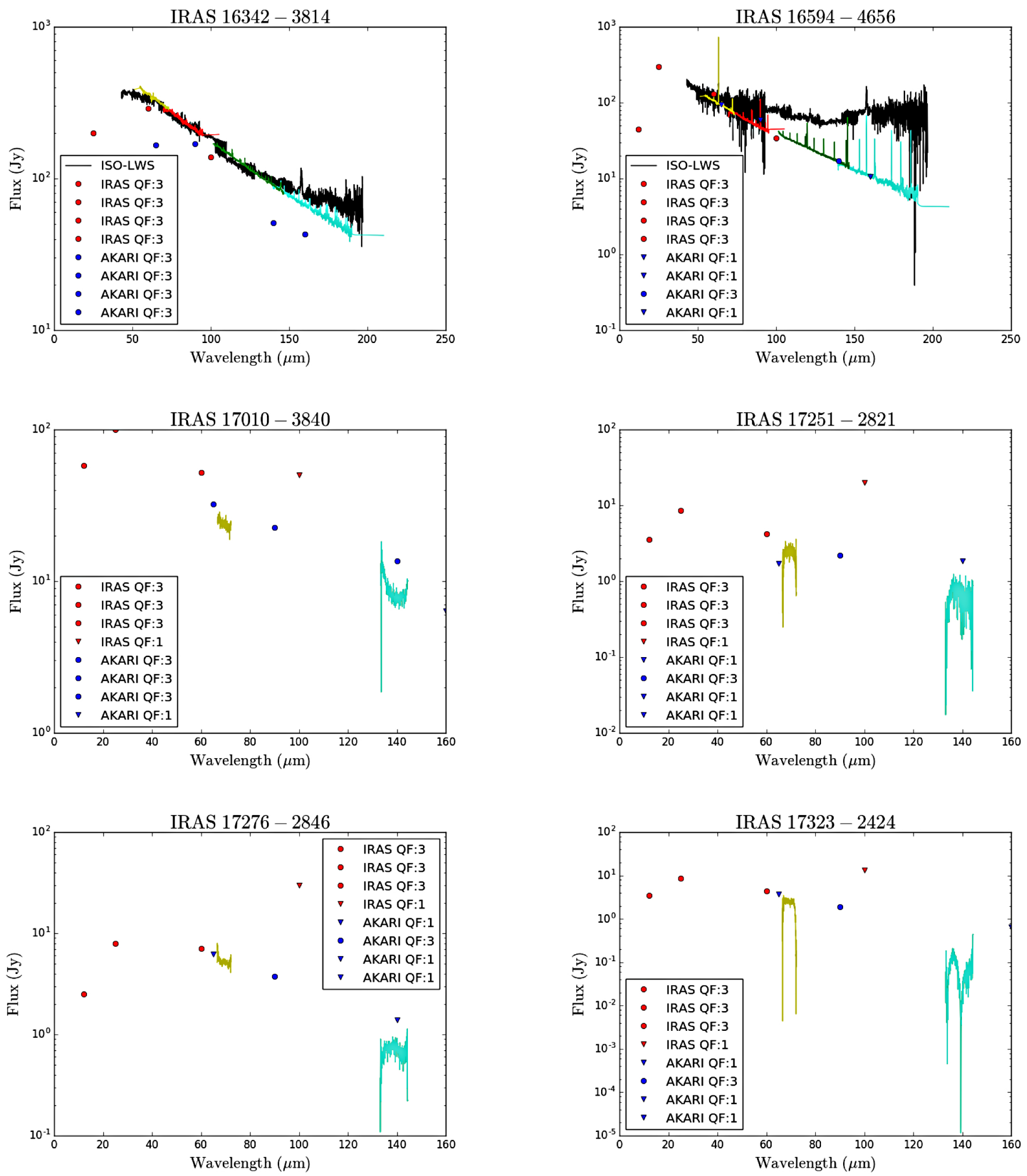

Fig. A.1. continued. 

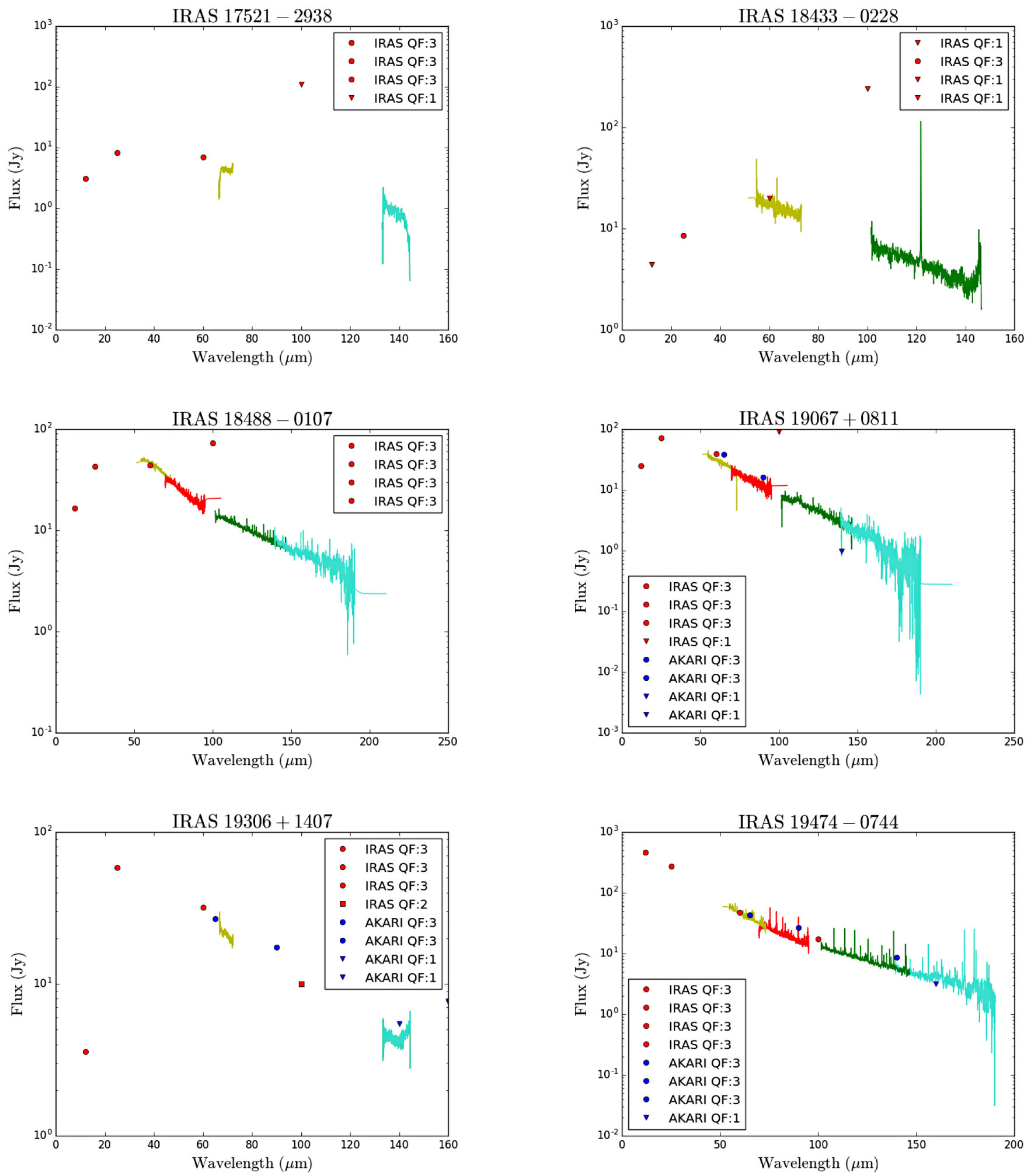

Fig. A.1. continued. 

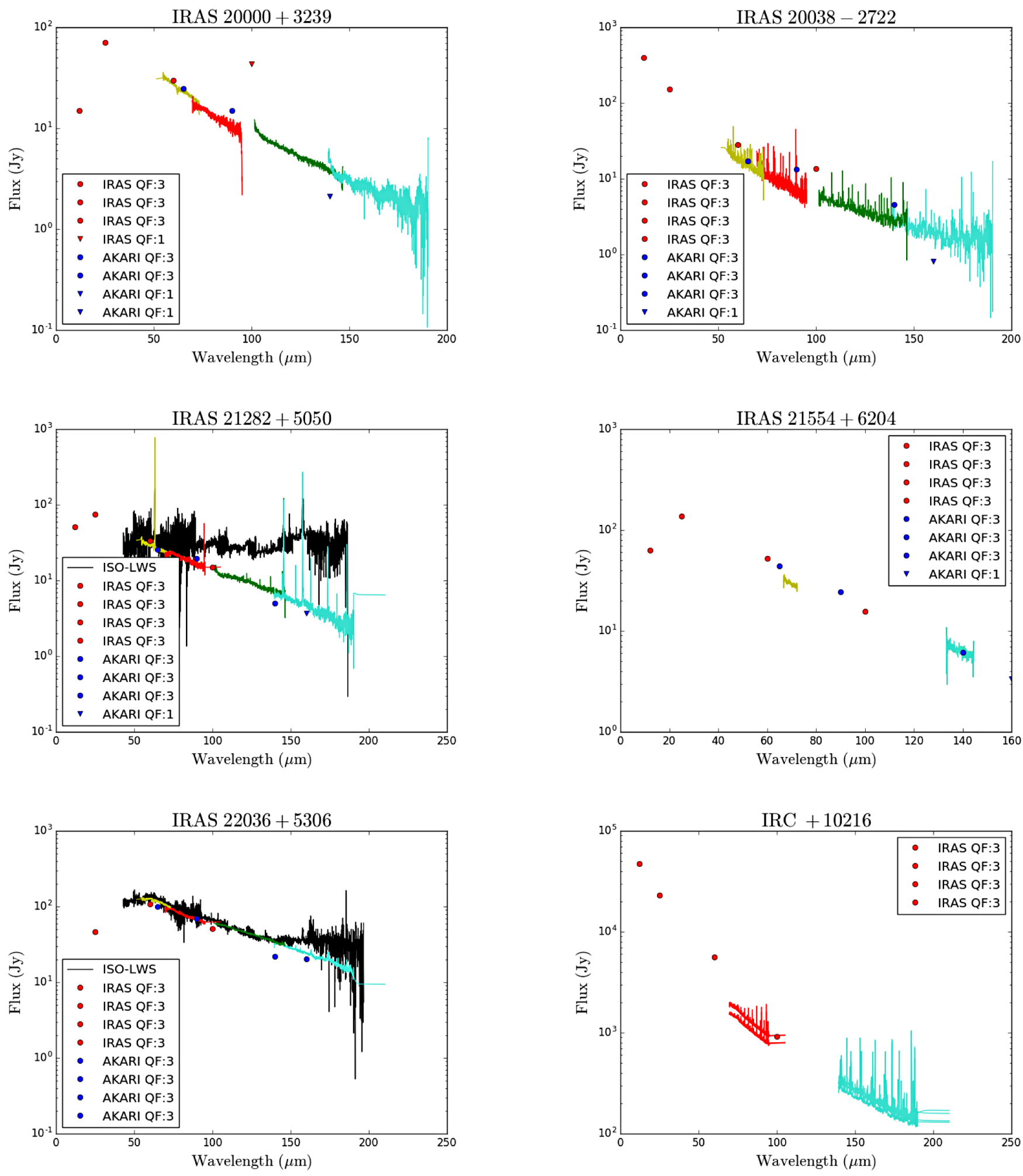

Fig. A.1. continued. 

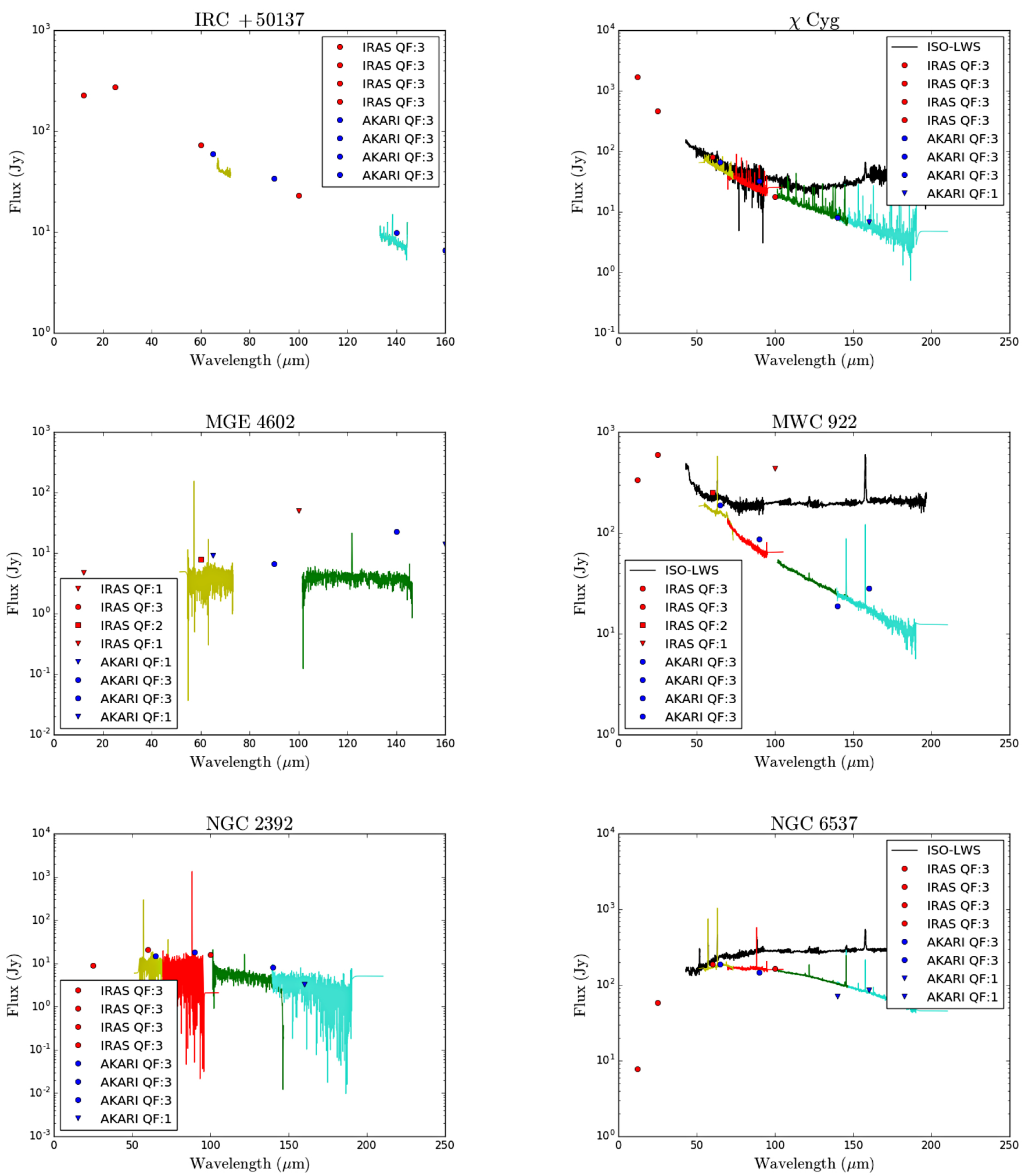

Fig. A.1. continued. 

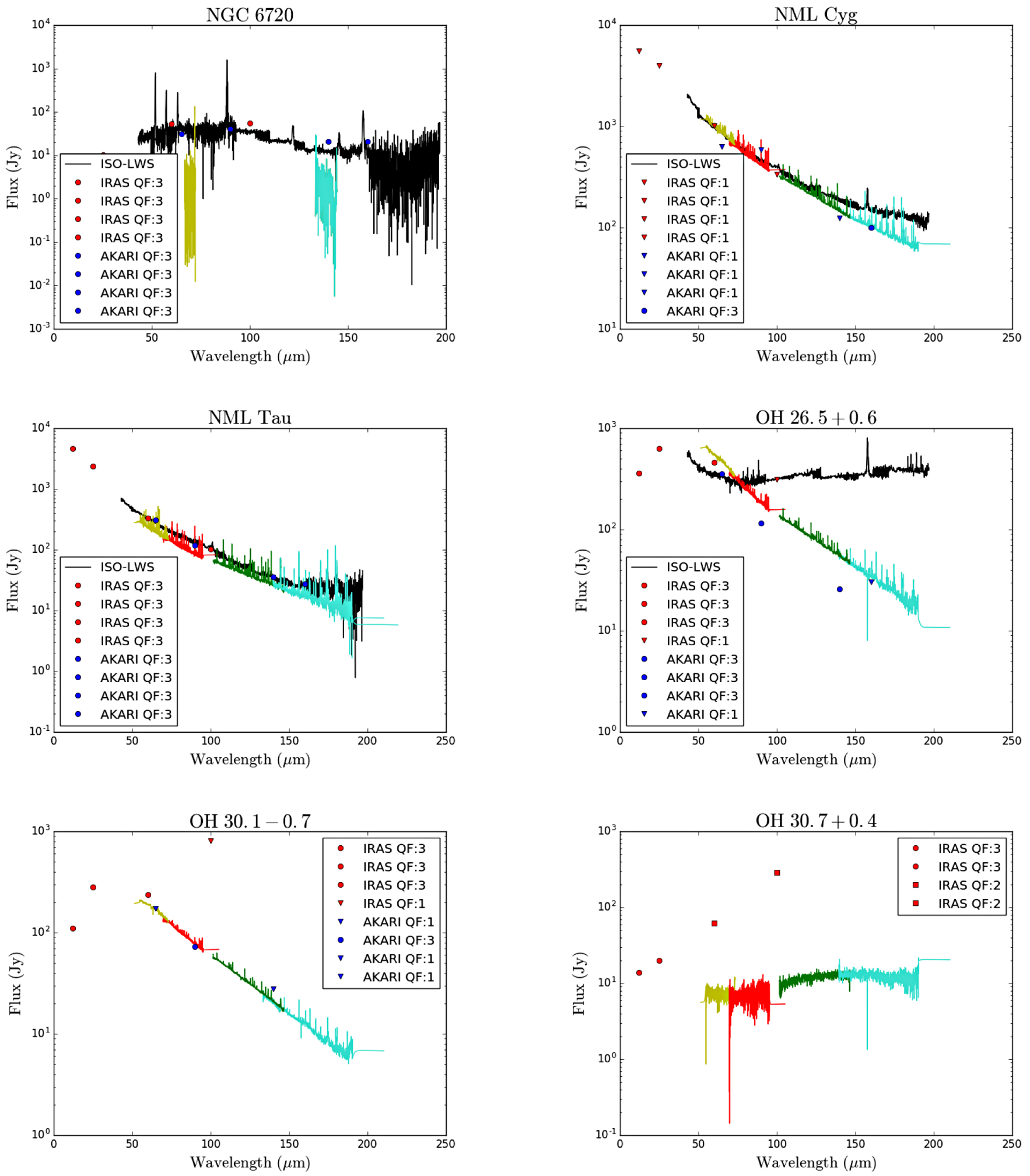

Fig. A.1. continued. 

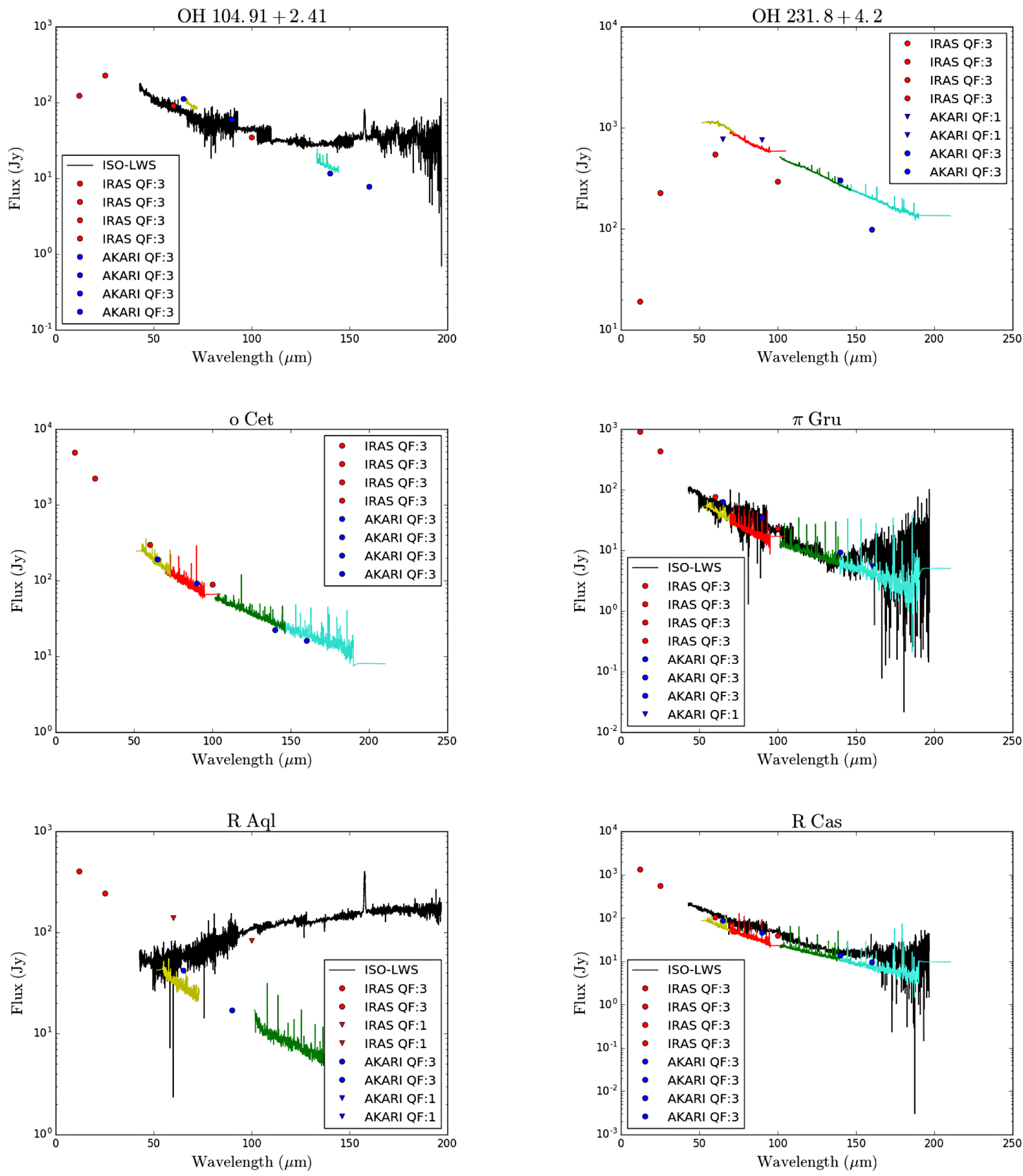

Fig. A.1. continued. 

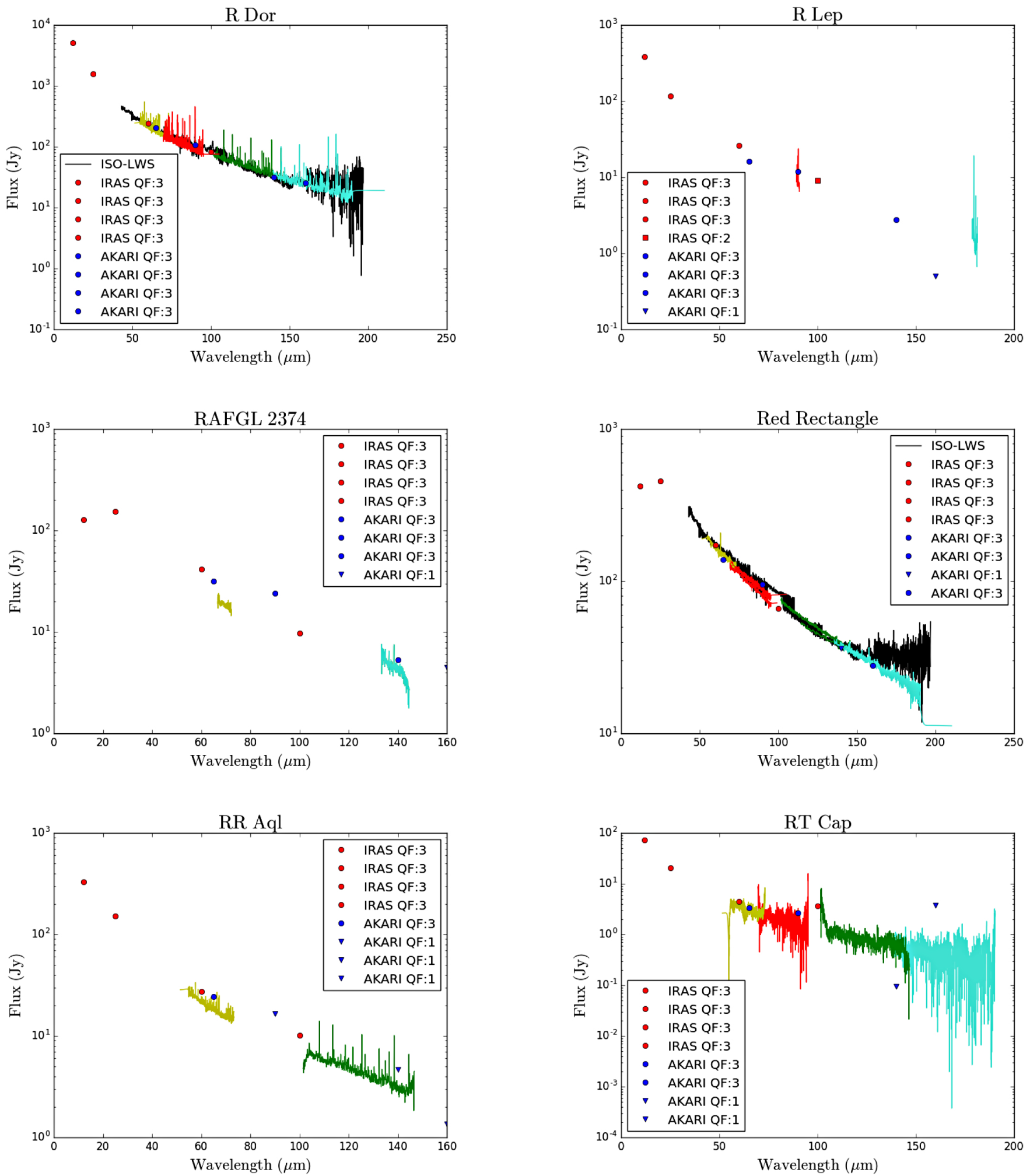

Fig. A.1. continued. 

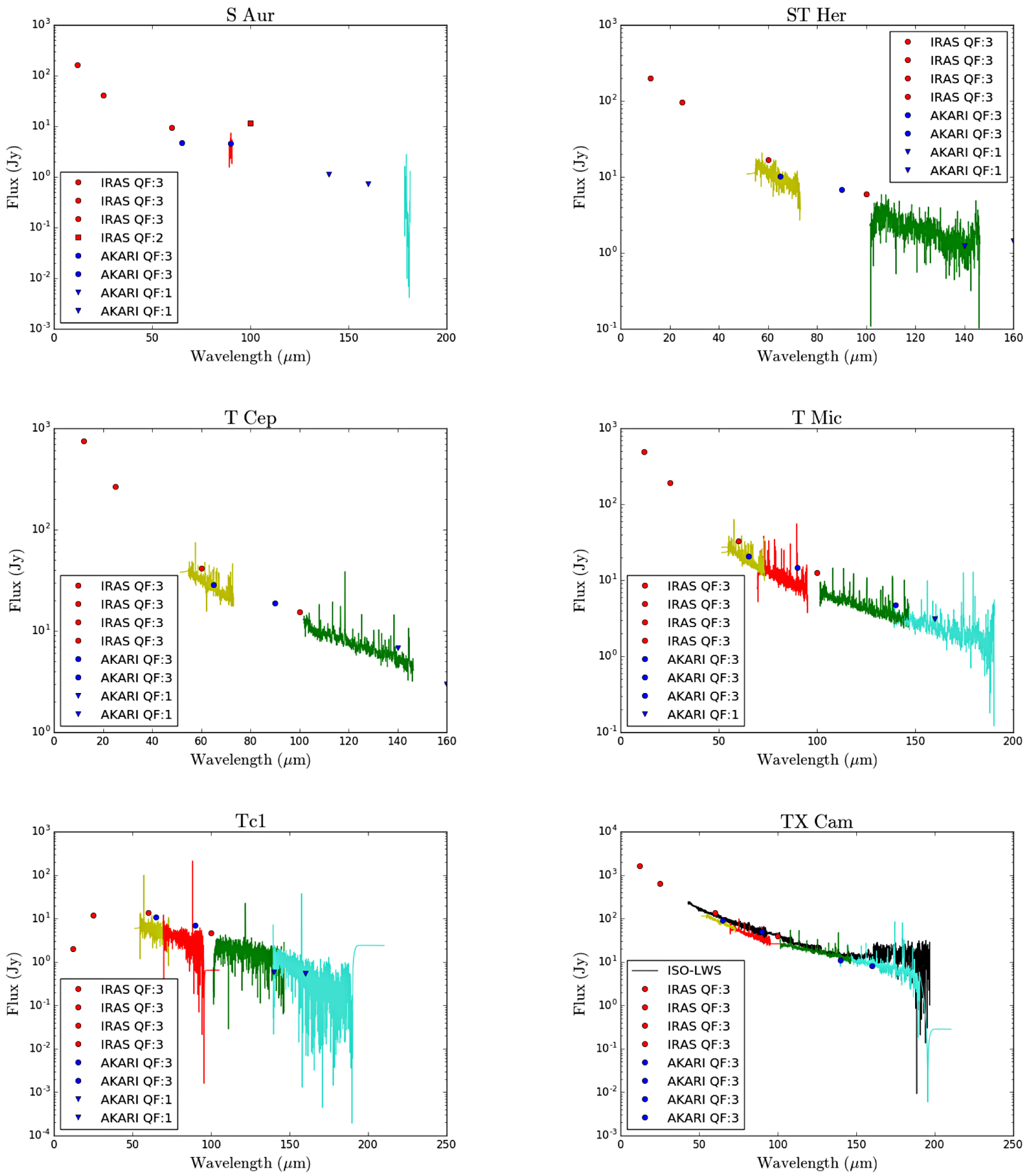

Fig. A.1. continued. 

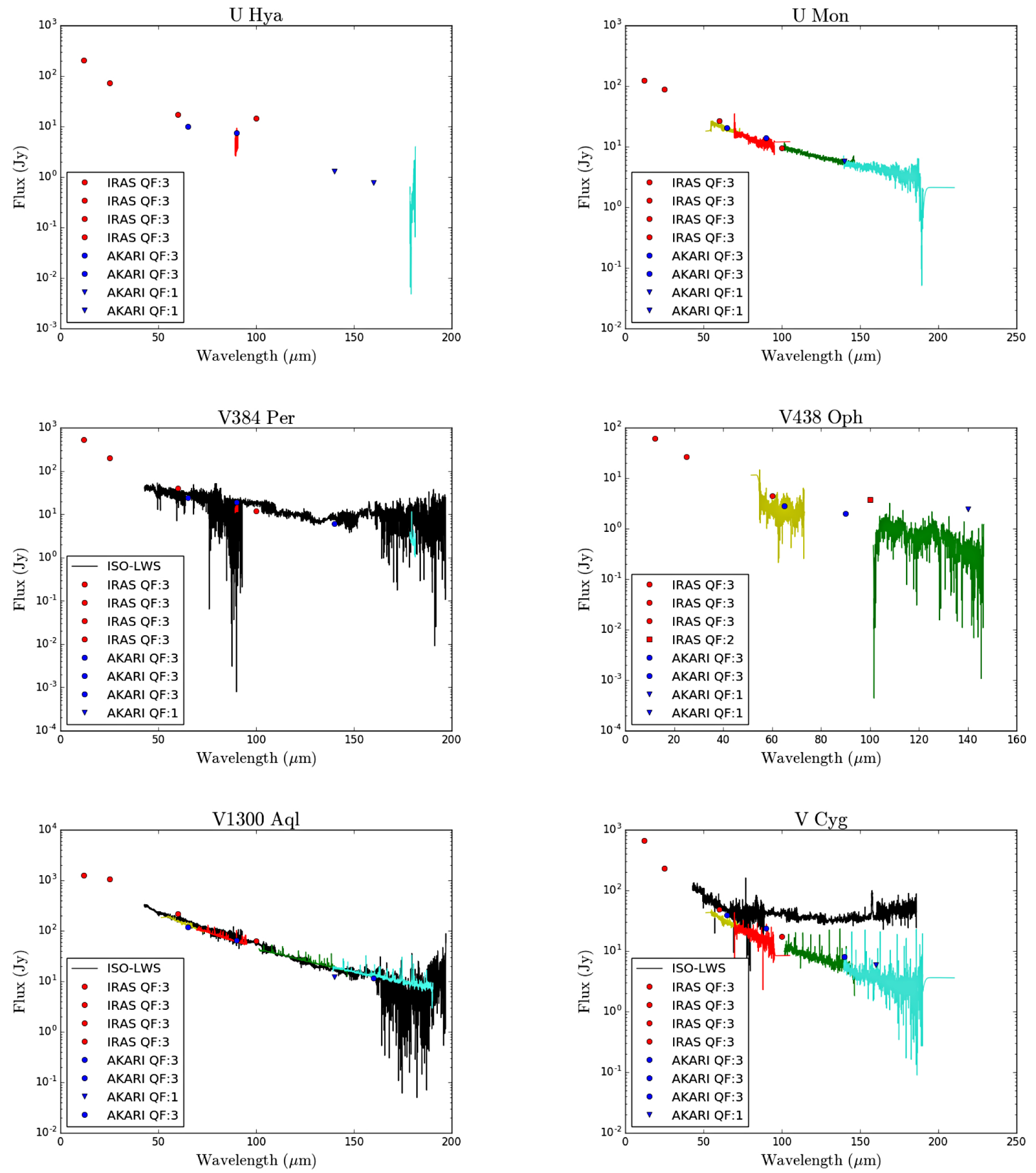

Fig. A.1. continued. 

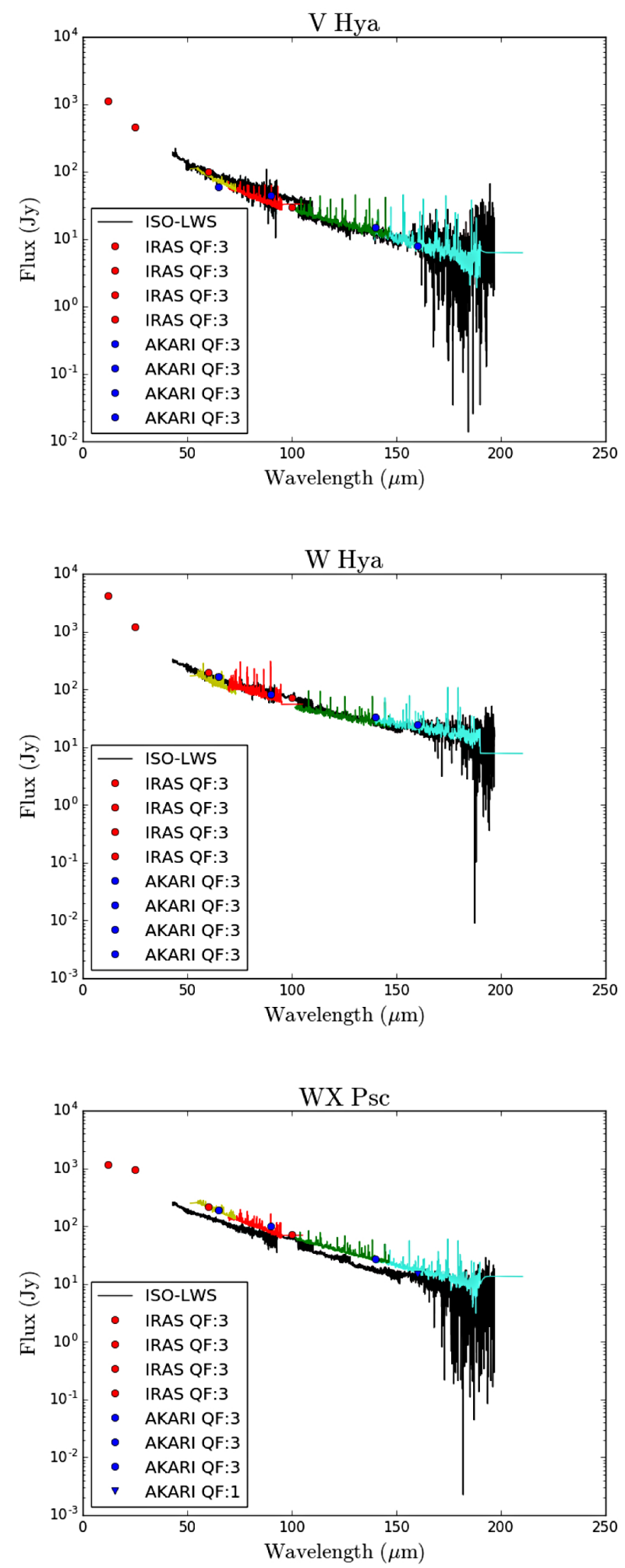

Fig. A.1. continued.
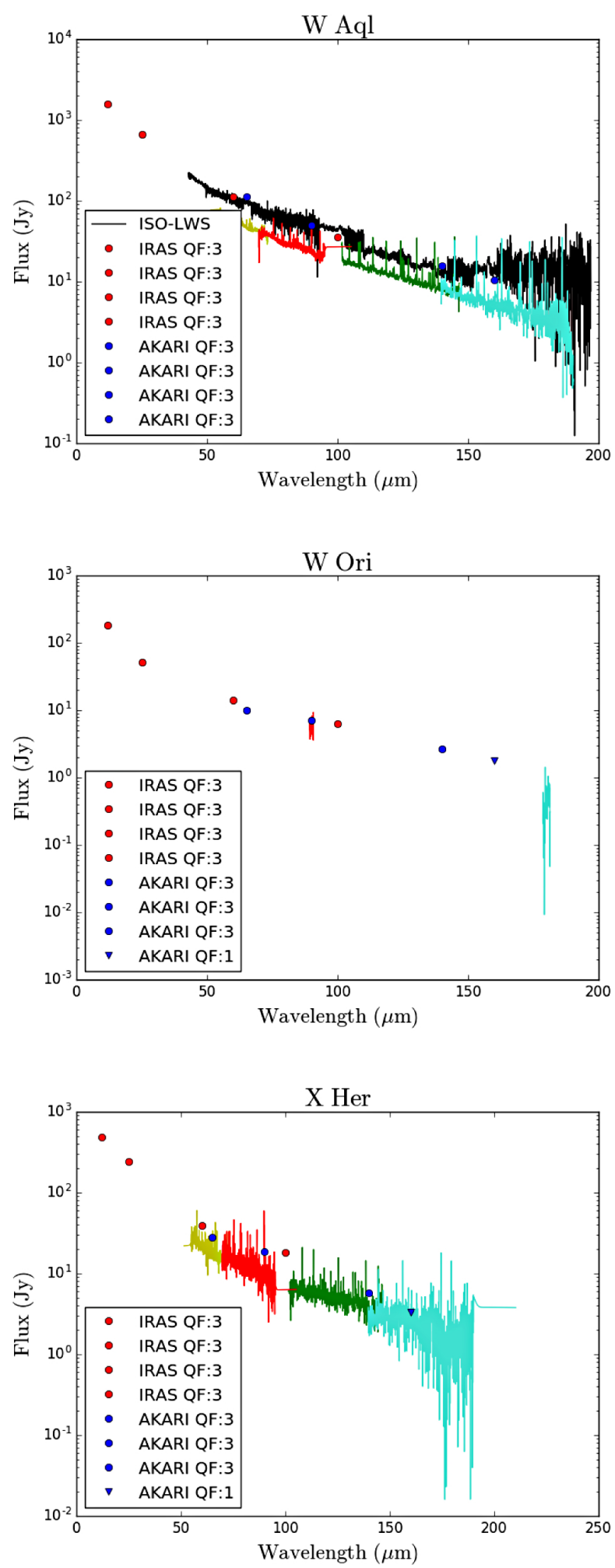
J. Ramos-Medina et al.: THROES: PACS spectroscopy

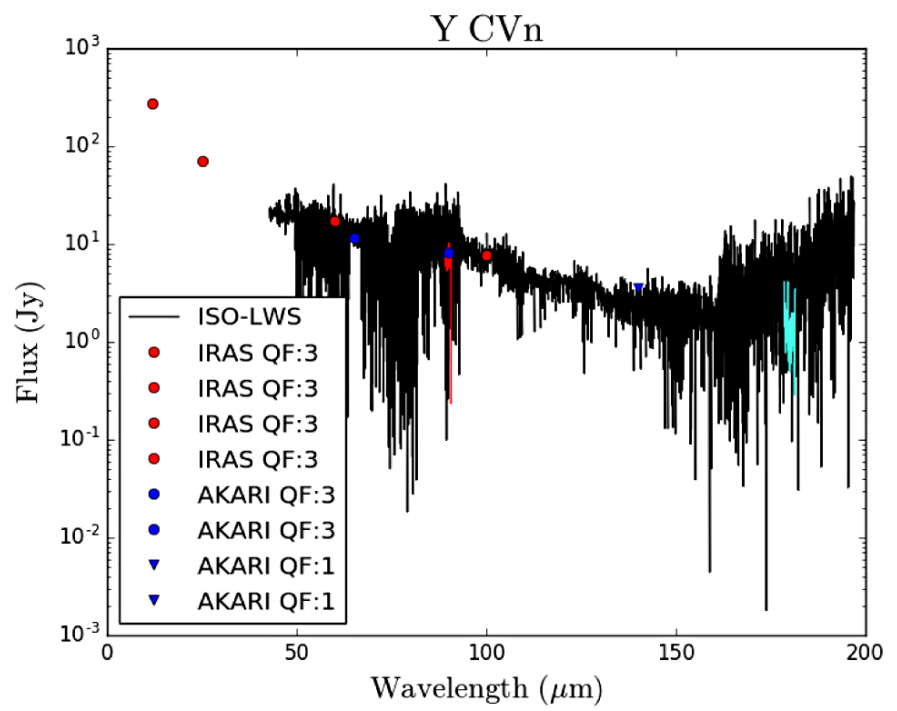

Fig. A.1. continued. 

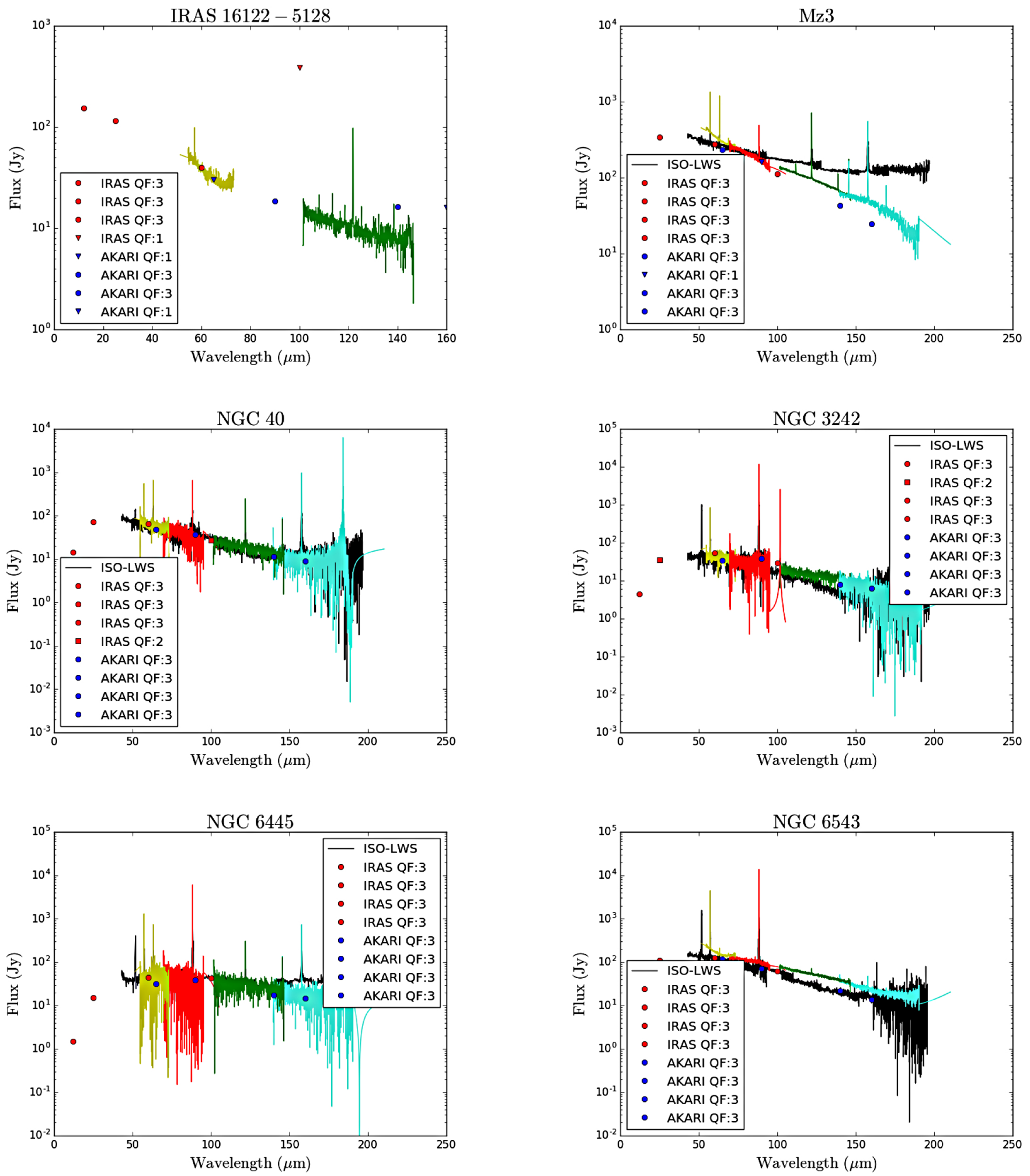

Fig. A.2. As in Fig. A.1 but for extended objects after applying point source flux loss correction and extended $55 \times 5$ correction. IRAS $16122-5128$ appears in THROES catalogue and in HSA as MGE 4218. 
J. Ramos-Medina et al.: THROES: PACS spectroscopy
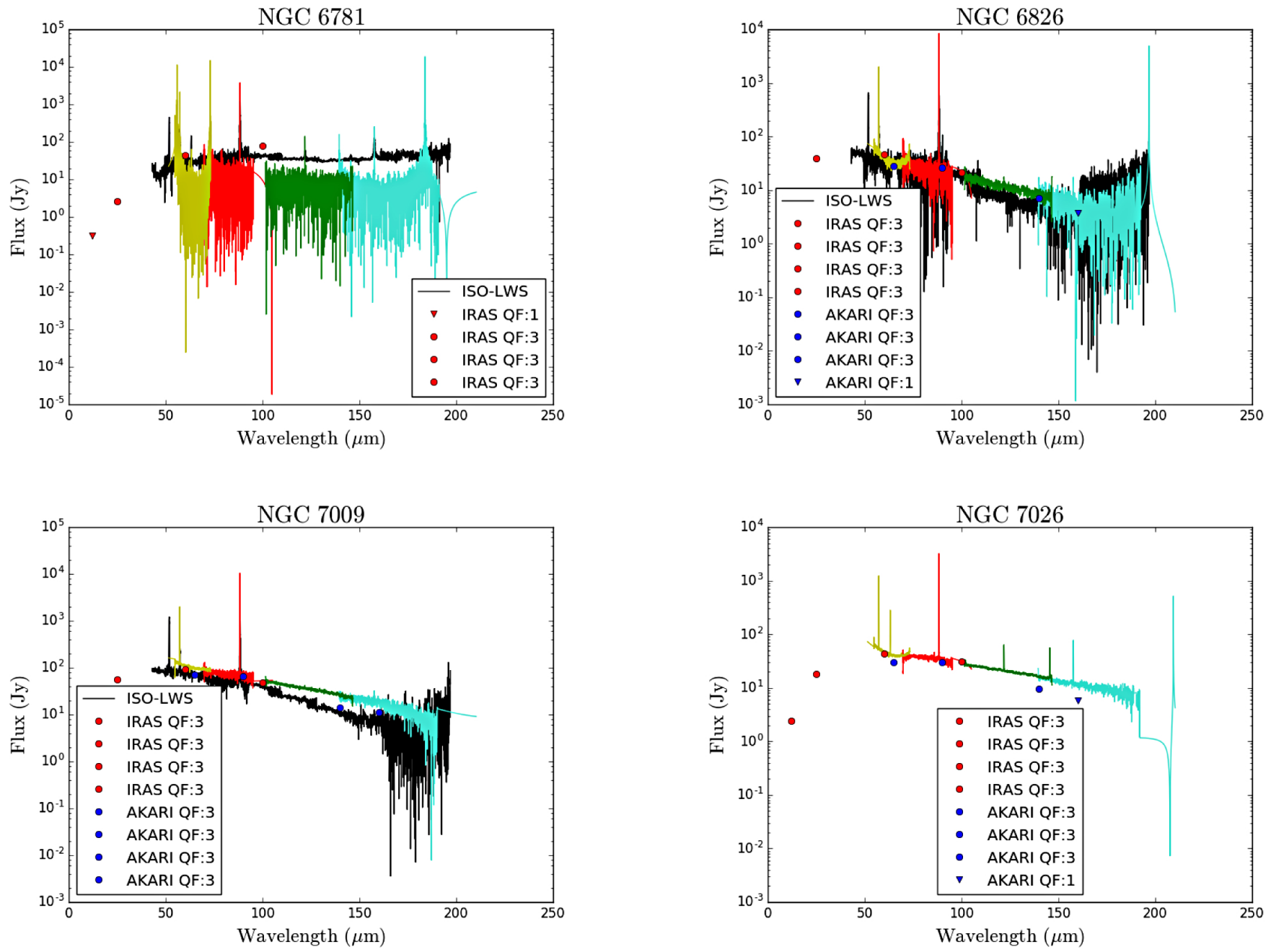

Fig. A.2. continued. 

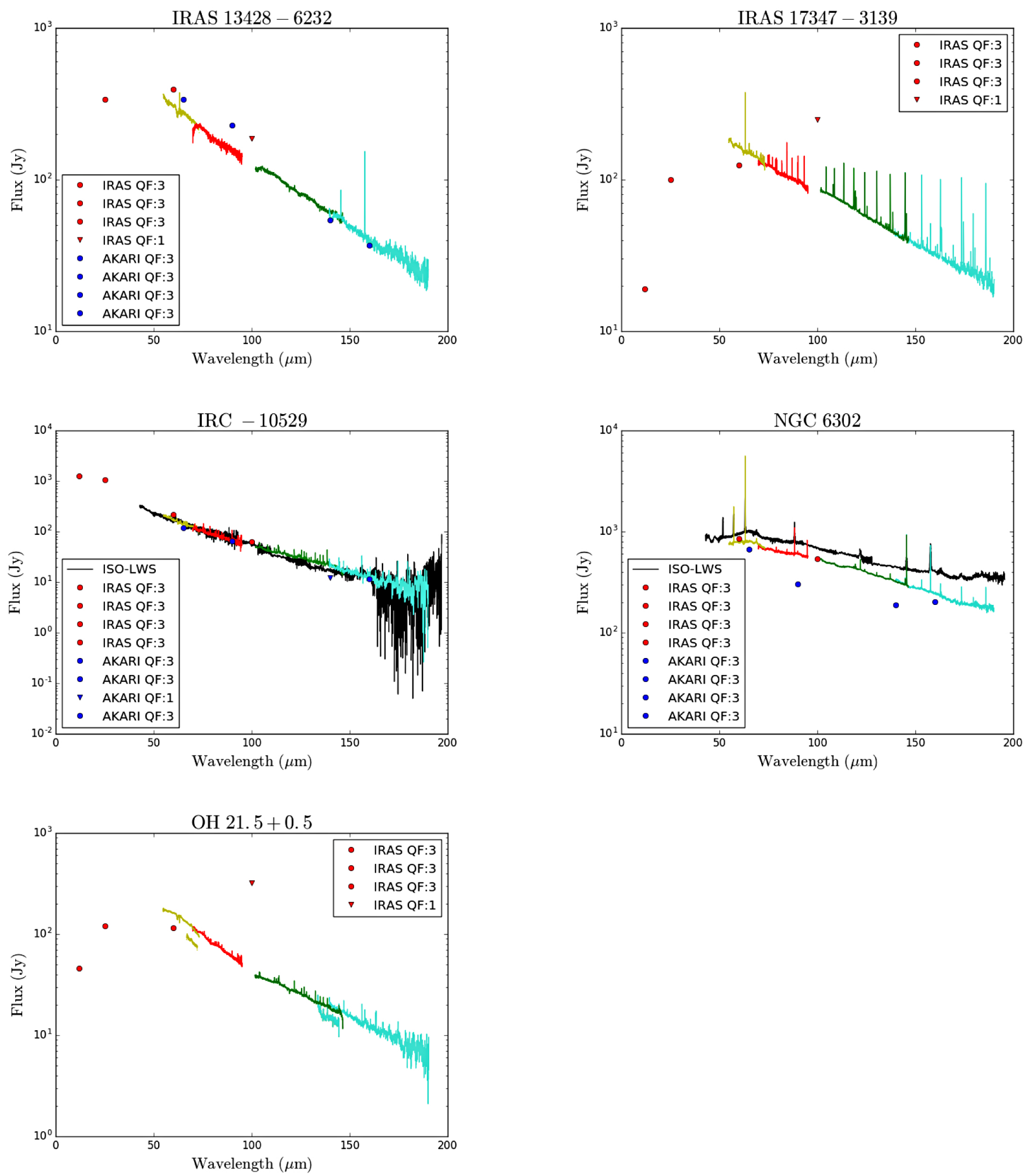

Fig. A.3. As in Fig. A.1 but for mispointed sources after applying point sources correction and semi-extended $3 \times 3$ correction. 

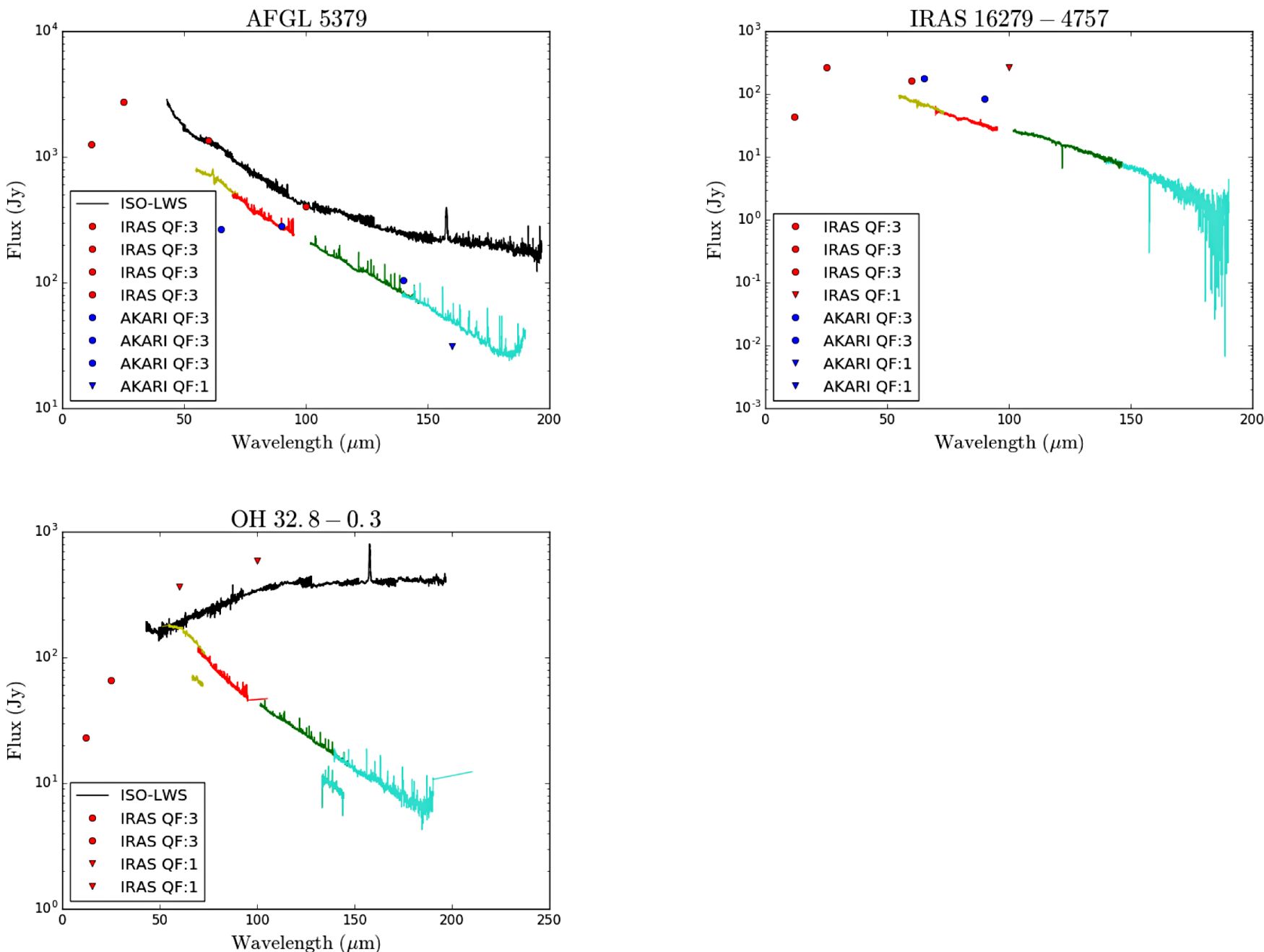

Fig. A.4. As in Fig. A.1 but for those sources corrected only by point sources correction. AFGL 5379 and IRAS 16279-4757 are mispointed targets for which the semi-extended $3 \times 3$ correction could not be applied as they are located in one of the outermost spaxels. The well-pointed object $\mathrm{OH} 32.8-0.3$ shows, for unknown reasons probably related to problems during the observations, negative fluxes in some of the $3 \times 3$ central spaxels, so the semi-extended $3 \times 3$ correction produced a wrong final $1 \mathrm{D}$ spectrum. 
Table A.1. Basic information about all observations reprocessed in THROES.

\begin{tabular}{|c|c|c|c|c|c|c|c|c|}
\hline Target name & RA (deg) & Dec (deg) & IRAS & AKARI & ISO & Comments & ObsIDs & Date \\
\hline AC Her & 277.5676 & 21.8668 & Yes & Yes & Yes & 1,6 & 1342208896 & 2010-11-13 \\
\hline AFGL 618 & 70.7236 & 36.1147 & Yes & Yes & Yes & - & $\begin{array}{l}1342225838 \\
1342225839 \\
1342225840 \\
\end{array}$ & $\begin{array}{l}2011-08-07 \\
2011-08-07 \\
2011-08-07 \\
\end{array}$ \\
\hline AFGL 2019 & 268.3282 & -26.9436 & Yes & Yes & No & 1,6 & 1342252253 & 2012-10-05 \\
\hline AFGL 2403 & 292.6228 & 19.8447 & Yes & Yes & No & 1 & 1342245226 & 2012-05-01 \\
\hline AFGL 2513 & 302.3093 & 31.4291 & Yes & Yes & No & - & $\begin{array}{l}1342270010 \\
1342269936 \\
\end{array}$ & $\begin{array}{l}2013-04-14 \\
2013-04-12 \\
\end{array}$ \\
\hline AFGL 2688 & 315.5781 & 36.6938 & No & No & Yes & - & $\begin{array}{l}1342199233 \\
1342199234 \\
1342199235 \\
\end{array}$ & $\begin{array}{l}2010-06-26 \\
2010-06-26 \\
2010-06-27\end{array}$ \\
\hline AFGL 3068 & 349.8016 & 17.1931 & Yes & Yes & Yes & - & $\begin{array}{l}1342199417 \\
1342199418 \\
1342257635\end{array}$ & $\begin{array}{l}2010-06-30 \\
2010-06-30 \\
2012-12-21\end{array}$ \\
\hline AFGL 3116 & 353.6152 & 43.5506 & Yes & Yes & No & - & $\begin{array}{l}1342212512 \\
1342212513\end{array}$ & $\begin{array}{l}2011-01-11 \\
2011-01-11 \\
\end{array}$ \\
\hline AFGL 4106 & 155.8311 & -59.5346 & Yes & Yes & Yes & 1 & 1342207818 & 2010-11-03 \\
\hline AFGL 4202 & 223.1012 & -62.0721 & Yes & Yes & No & 1,6 & 1342250004 & 2012-08-21 \\
\hline AFGL 4259 & 301.5947 & 27.0362 & Yes & Yes & No & 1,6 & 1342244918 & 2012-04-24 \\
\hline AFGL 5379 & 266.0942 & -31.9276 & Yes & Yes & Yes & 2 & $\begin{array}{l}1342228537 \\
1342228538 \\
\end{array}$ & $\begin{array}{l}2011-09-13 \\
2011-09-13 \\
\end{array}$ \\
\hline AFGL 6815 & 259.5830 & -32.4556 & Yes & Yes & No & - & $\begin{array}{l}1342216629 \\
1342216630\end{array}$ & $\begin{array}{l}2011-03-22 \\
2011-03-22\end{array}$ \\
\hline AQ Sgr & 293.5791 & -16.3741 & Yes & Yes & No & - & $\begin{array}{l}1342268751 \\
1342268752 \\
\end{array}$ & $\begin{array}{l}2013-03-28 \\
2013-03-28 \\
\end{array}$ \\
\hline $\mathrm{BD}+303639$ & 293.6884 & 30.5163 & Yes & Yes & Yes & 3 & $\begin{array}{l}1342220600 \\
1342220601\end{array}$ & $\begin{array}{l}2011-05-04 \\
2011-05-04 \\
\end{array}$ \\
\hline CIT 6 & 154.0094 & 30.5718 & Yes & No & Yes & - & $\begin{array}{l}1342197799 \\
1342197800 \\
\end{array}$ & $\begin{array}{l}2010-06-05 \\
2010-06-05 \\
\end{array}$ \\
\hline CPD-568032 & 257.2536 & -56.9133 & Yes & Yes & Yes & - & $\begin{array}{l}1342228201 \\
1342228202 \\
\end{array}$ & $\begin{array}{l}2011-09-06 \\
2011-09-06 \\
\end{array}$ \\
\hline CPD-642939 & 219.2920 & -64.8013 & Yes & Yes & No & 1,6 & 1342250006 & 2012-08-21 \\
\hline EP Aqr & 326.6327 & -2.2127 & Yes & Yes & No & - & $\begin{array}{l}1342270639 \\
1342270684\end{array}$ & $\begin{array}{l}2013-04-20 \\
2013-04-21 \\
\end{array}$ \\
\hline G Her & 247.1606 & 41.8816 & Yes & Yes & No & 1 & 1342247780 & 2012-07-09 \\
\hline HD 56126 & 109.0427 & 9.9966 & Yes & Yes & Yes & - & $\begin{array}{l}1342220930 \\
1342220931 \\
\end{array}$ & $\begin{array}{l}2011-04-30 \\
2011-04-30 \\
\end{array}$ \\
\hline HD 161796 & 266.2311 & 50.0443 & Yes & Yes & Yes & - & $\begin{array}{l}1342208881 \\
1342208882 \\
\end{array}$ & $\begin{array}{l}2010-11-12 \\
2010-11-12 \\
\end{array}$ \\
\hline HD 235858 & 337.2932 & 54.8517 & Yes & Yes & No & - & $\begin{array}{l}1342196686 \\
1342196687\end{array}$ & $\begin{array}{l}2010-05-18 \\
2010-05-18\end{array}$ \\
\hline HD 331319 & 297.3731 & 31.4545 & Yes & Yes & No & 1 & 1342245232 & 2012-05-01 \\
\hline Hen 2-90 & 197.4009 & -61.3266 & Yes & Yes & Yes & 1,6 & 1342248308 & 2012-07-16 \\
\hline
\end{tabular}

Notes. Column 1: target name; Cols. 2 and 3: equatorial coordinates (J2000) in degrees; Cols. 4-6: complementary data for each observation in IRAS, AKARI, or ISO; Col. 7: comments on complementary information about the PACS observations object: 1) incomplete PACS wavelength coverage; 2) mispointing (target not in the central spaxel); 3) semi-extended object (size $\sim 3 \times 3$ central spaxels); 4) extended object (size $\sim 5 \times$ 5 spaxels); 5) contamination in ISO data due to interstellar emission; 6) faint source ( $\leq 10 \mathrm{Jy}$ at $60 \mu \mathrm{m}$ ); 7) multi epochs observations. If the observation presents a complete spectral coverage in PACS wavelength range, is well pointed, Chop/Nod and the source is point-like and intense, there is no comment in the column (-); Col. 8: ObsIds; Col. 9: date of observation. 
Table A.1. continued.

\begin{tabular}{|c|c|c|c|c|c|c|c|c|}
\hline Target name & RA (deg) & Dec (deg) & IRAS & AKARI & ISO & Comments & ObsIDs & Date \\
\hline Hen 2-113 & 224.9730 & -54.3020 & Yes & Yes & Yes & - & $\begin{array}{l}1342225142 \\
1342225143 \\
1342249211 \\
\end{array}$ & $\begin{array}{l}2011-08-02 \\
2011-08-02 \\
2012-08-07\end{array}$ \\
\hline Hen 3-401 & 154.8852 & -60.2248 & Yes & Yes & No & - & $\begin{array}{l}1342225588 \\
1342225589 \\
\end{array}$ & $\begin{array}{l}2011-08-03 \\
2011-08-03 \\
\end{array}$ \\
\hline Hen 3-1475 & 266.3090 & -17.9462 & Yes & Yes & Yes & - & $\begin{array}{l}1342229719 \\
1342229720 \\
\end{array}$ & $\begin{array}{l}2011-09-24 \\
2011-09-24 \\
\end{array}$ \\
\hline IC 418 & 81.8675 & -12.6973 & Yes & No & Yes & 3 & $\begin{array}{l}1342265942 \\
1342265943\end{array}$ & $\begin{array}{l}2013-03-04 \\
2013-03-04\end{array}$ \\
\hline IRAS 07027-7934 & 104.8599 & -79.6463 & Yes & Yes & Yes & 1,6 & 1342245248 & 2012-05-02 \\
\hline IRAS 08011-3627 & 120.7568 & -36.5966 & Yes & Yes & No & - & $\begin{array}{l}1342210381 \\
1342256781 \\
1342256782 \\
\end{array}$ & $\begin{array}{l}2010-11-27 \\
2012-12-04 \\
2012-12-04\end{array}$ \\
\hline IRAS 08544-4431 & 134.0591 & -44.7196 & Yes & Yes & No & - & $\begin{array}{l}1342245956 \\
1342245957 \\
\end{array}$ & $\begin{array}{l}2012-05-21 \\
2012-05-21 \\
\end{array}$ \\
\hline IRAS 09256-6324 & 141.7220 & -63.6302 & Yes & Yes & No & - & $\begin{array}{l}1342210386 \\
1342248357 \\
1342248358 \\
\end{array}$ & $\begin{array}{l}2010-11-27 \\
2012-07-20 \\
2012-07-20 \\
\end{array}$ \\
\hline IRAS $09371+1212$ & 144.9749 & 11.9814 & Yes & Yes & No & - & $\begin{array}{l}1342245647 \\
1342245648 \\
\end{array}$ & $\begin{array}{l}2012-05-12 \\
2012-05-12 \\
\end{array}$ \\
\hline IRAS 09425-6040 & 146.0066 & -60.9072 & Yes & Yes & No & - & $\begin{array}{l}1342225563 \\
1342225564 \\
\end{array}$ & $\begin{array}{l}2011-07-25 \\
2011-07-25 \\
\end{array}$ \\
\hline IRAS 10456-5712 & 161.91 & -57.4674 & Yes & Yes & No & - & $\begin{array}{l}1342211823 \\
1342248928 \\
1342248929 \\
\end{array}$ & $\begin{array}{l}2010-12-28 \\
2012-07-31 \\
2012-07-31 \\
\end{array}$ \\
\hline IRAS 13428-6232 & 206.5854 & -62.8000 & Yes & Yes & No & 2 & $\begin{array}{l}1342212212 \\
1342212213\end{array}$ & $\begin{array}{l}2010-12-31 \\
2010-12-31 \\
\end{array}$ \\
\hline IRAS 15194-5115 & 230.7704 & -51.4330 & Yes & Yes & Yes & - & $\begin{array}{l}1342215685 \\
1342215686\end{array}$ & $\begin{array}{l}2011-03-10 \\
2011-03-10 \\
\end{array}$ \\
\hline IRAS 16122-5128 & 244.0190 & -51.5989 & Yes & Yes & No & 1 & 1342240167 & 2012-02-18 \\
\hline IRAS 16279-4757 & 247.9087 & -48.0677 & Yes & Yes & No & 2 & $\begin{array}{l}1342228203 \\
1342228204 \\
\end{array}$ & $\begin{array}{l}2011-09-06 \\
2011-09-06 \\
\end{array}$ \\
\hline IRAS 16342-3814 & 249.417 & -38.3380 & Yes & Yes & Yes & - & $\begin{array}{l}1342216627 \\
1342216628 \\
\end{array}$ & $\begin{array}{l}2011-03-22 \\
2011-03-22 \\
\end{array}$ \\
\hline IRAS 16594-4656 & 255.7917 & -47.0076 & Yes & Yes & Yes & 5 & $\begin{array}{l}1342228414 \\
1342228415 \\
\end{array}$ & $\begin{array}{l}2011-09-10 \\
2011-09-10 \\
\end{array}$ \\
\hline IRAS 17010-3840 & 256.1179 & -38.7396 & Yes & Yes & No & 1,6 & 1342216176 & 2011-03-16 \\
\hline IRAS 17251-2821 & 262.0770 & -28.3991 & Yes & Yes & No & 1,6 & 1342228535 & 2011-09-13 \\
\hline IRAS 17276-2846 & 262.7012 & -28.8172 & Yes & Yes & No & 1,6 & 1342228536 & 2011-09-13 \\
\hline IRAS 17323-2424 & 263.8583 & -24.4422 & Yes & Yes & No & 1,6 & 1342228532 & 2011-09-13 \\
\hline IRAS 17347-3139 & 264.5054 & -31.6827 & Yes & No & No & 2 & $\begin{array}{l}1342229696 \\
1342229697\end{array}$ & $\begin{array}{l}2011-09-24 \\
2011-09-24\end{array}$ \\
\hline IRAS 17521-2938 & 268.8404 & -29.6536 & Yes & No & No & 1,6 & 1342229802 & 2011-09-27 \\
\hline IRAS 18433-0228 & 281.4800 & -2.4189 & Yes & No & No & 1 & 1342231743 & 2011-11-01 \\
\hline IRAS 18488-0107 & 282.8592 & -1.0645 & Yes & No & No & - & $\begin{array}{l}1342268791 \\
1342268792 \\
\end{array}$ & $\begin{array}{l}2013-03-30 \\
2013-03-30 \\
\end{array}$ \\
\hline IRAS 19067+0811 & 287.2845 & 8.2770 & Yes & Yes & No & - & $\begin{array}{l}1342268797 \\
1342268798 \\
\end{array}$ & $\begin{array}{l}2013-03-31 \\
2013-03-31 \\
\end{array}$ \\
\hline IRAS 19306+1407 & 293.2295 & 14.2269 & Yes & Yes & No & 1,6 & 1342244921 & 2012-04-24 \\
\hline IRAS 19474-0744 & 297.5264 & -7.6145 & Yes & Yes & No & - & $\begin{array}{l}1342268638 \\
1342268449\end{array}$ & $\begin{array}{l}2013-03-25 \\
2013-03-26\end{array}$ \\
\hline
\end{tabular}


Table A.1. continued.

\begin{tabular}{|c|c|c|c|c|c|c|c|c|}
\hline Target name & RA (deg) & Dec (deg) & IRAS & AKARI & ISO & Comments & ObsIDs & Date \\
\hline IRAS $20000+3239$ & 300.4980 & 32.7924 & Yes & Yes & No & - & $\begin{array}{l}1342270612 \\
1342270344 \\
\end{array}$ & $\begin{array}{l}2013-04-18 \\
2013-04-17 \\
\end{array}$ \\
\hline IRAS 20038-2722 & 301.7301 & -27.2249 & Yes & Yes & No & - & $\begin{array}{l}1342268730 \\
1342268569 \\
\end{array}$ & $\begin{array}{l}2013-03-29 \\
2013-03-27 \\
\end{array}$ \\
\hline IRAS $21282+5050$ & 322.4934 & 51.0666 & Yes & Yes & Yes & 5 & $\begin{array}{l}1342223375 \\
1342220741 \\
\end{array}$ & $\begin{array}{l}2011-06-30 \\
2011-05-12 \\
\end{array}$ \\
\hline IRAS 21554+6204 & 329.2424 & 62.3121 & Yes & Yes & No & 1,6 & $\begin{array}{l}1342245813 \\
1342247150 \\
\end{array}$ & $\begin{array}{l}2012-05-15 \\
2012-06-20 \\
\end{array}$ \\
\hline IRAS $22036+5306$ & 331.3761 & 53.3591 & Yes & Yes & Yes & 5 & $\begin{array}{l}1342221882 \\
1342221883\end{array}$ & $\begin{array}{l}2011-05-29 \\
2011-05-29\end{array}$ \\
\hline IRC + 10216 & 146.9892 & 13.2787 & Yes & No & No & 1,7 & $\begin{array}{l}1342245395 \\
1342221889 \\
1342253754 \\
1342256262 \\
\end{array}$ & $\begin{array}{l}2012-05-05 \\
2011-05-29 \\
2012-10-21 \\
2012-11-30 \\
\end{array}$ \\
\hline IRC $-10529^{13}$ & 302.6142 & -6.2710 & Yes & Yes & Yes & 2 & $\begin{array}{l}1342208931 \\
1342208932 \\
\end{array}$ & $\begin{array}{l}2010-11-14 \\
2010-11-14 \\
\end{array}$ \\
\hline IRC +50137 & 77.831 & 52.8758 & Yes & Yes & No & 1 & 1342249315 & 2012-08-10 \\
\hline
\end{tabular}

\begin{tabular}{|c|c|c|c|c|c|c|c|c|}
\hline$\chi \mathrm{Cyg}$ & 297.6413 & 32.9140 & Yes & Yes & Yes & - & $\begin{array}{l}1342198176 \\
1342198177 \\
\end{array}$ & $\begin{array}{l}2010-06-02 \\
2010-06-02 \\
\end{array}$ \\
\hline MGE 4602 & 271.1620 & -20.6242 & Yes & Yes & No & 1,6 & 1342243506 & 2012-03-24 \\
\hline MWC 922 & 275.3162 & -13.0241 & Yes & Yes & Yes & 5 & $\begin{array}{l}1342229805 \\
1342229806 \\
\end{array}$ & $\begin{array}{l}2011-09-27 \\
2011-09-27\end{array}$ \\
\hline Mz 3 & 244.3058 & -51.9862 & Yes & Yes & Yes & 4 & $\begin{array}{l}1342243109 \\
1342243110 \\
\end{array}$ & $\begin{array}{l}2012-03-21 \\
2012-03-21 \\
\end{array}$ \\
\hline NGC 40 & 3.2542 & 72.5219 & Yes & Yes & Yes & 4,6 & $\begin{array}{l}1342236879 \\
1342236880 \\
\end{array}$ & $\begin{array}{l}2012-01-08 \\
2012-01-08 \\
\end{array}$ \\
\hline NGC 2392 & 112.2948 & 20.9118 & Yes & Yes & No & 6 & $\begin{array}{l}1342229792 \\
1342229816 \\
\end{array}$ & $\begin{array}{l}2011-09-26 \\
2011-09-27 \\
\end{array}$ \\
\hline NGC 3242 & 156.1920 & -18.6411 & Yes & Yes & Yes & 4,6 & $\begin{array}{l}1342232278 \\
1342232279 \\
\end{array}$ & $\begin{array}{l}2011-11-12 \\
2011-11-12 \\
\end{array}$ \\
\hline NGC 6302 & 258.4342 & -37.1044 & Yes & Yes & Yes & 2 & $\begin{array}{l}1342230150 \\
1342230151 \\
\end{array}$ & $\begin{array}{l}2011-10-05 \\
2011-10-05 \\
\end{array}$ \\
\hline NGC 6445 & 267.3133 & -20.0095 & Yes & Yes & Yes & 4,6 & $\begin{array}{l}1342242440 \\
1342242441 \\
\end{array}$ & $\begin{array}{l}2012-03-26 \\
2012-03-26 \\
\end{array}$ \\
\hline NGC 6537 & 271.3045 & -19.8430 & No & Yes & Yes & 3,5 & $\begin{array}{l}1342231322 \\
1342231323 \\
\end{array}$ & $\begin{array}{l}2011-10-22 \\
2011-10-22 \\
\end{array}$ \\
\hline NGC 6543 & 269.6385 & 66.6330 & No & Yes & Yes & 4 & $\begin{array}{l}1342238388 \\
1342238389 \\
1342212264 \\
\end{array}$ & $\begin{array}{l}2012-01-29 \\
2012-01-29 \\
2011-01-02\end{array}$ \\
\hline NGC 6543 WKnot & 269.5722 & 66.6356 & No & No & No & - & $\begin{array}{l}1342235679 \\
1342235680 \\
\end{array}$ & $\begin{array}{l}2011-12-27 \\
2011-12-27 \\
\end{array}$ \\
\hline NGC 6720 & 283.3961 & 33.0291 & Yes & Yes & Yes & 1,6 & 1342208920 & 2010-11-14 \\
\hline NGC 6720 OFFCenter & 283.3937 & 33.0326 & Yes & Yes & Yes & - & $\begin{array}{l}1342233716 \\
1342233717 \\
\end{array}$ & $\begin{array}{l}2011-12-07 \\
2011-12-07 \\
\end{array}$ \\
\hline NGC 6781 & 289.6170 & 6.5386 & Yes & No & Yes & 4,6 & $\begin{array}{l}1342230999 \\
1342231000 \\
\end{array}$ & $\begin{array}{l}2011-10-14 \\
2011-10-14 \\
\end{array}$ \\
\hline NGC 6781 Rim & 289.6313 & 6.5388 & No & No & No & - & $\begin{array}{l}1342231001 \\
1342231002 \\
\end{array}$ & $\begin{array}{l}2011-10-15 \\
2011-10-15 \\
\end{array}$ \\
\hline NGC 6826 & 296.2006 & 50.5250 & Yes & Yes & Yes & 3,6 & $\begin{array}{l}1342238926 \\
1342238927\end{array}$ & $\begin{array}{l}2012-02-10 \\
2012-02-10\end{array}$ \\
\hline
\end{tabular}


Table A.1. continued.

\begin{tabular}{|c|c|c|c|c|c|c|c|c|}
\hline Target name & RA (deg) & Dec (deg) & IRAS & AKARI & ISO & Comments & ObsIDs & Date \\
\hline NGC 6826 Rim & 296.2207 & 50.5290 & No & No & No & - & $\begin{array}{l}1342235850 \\
1342235851 \\
\end{array}$ & $\begin{array}{l}2011-12-31 \\
2011-12-31 \\
\end{array}$ \\
\hline NGC 7009 & 316.0450 & -11.3635 & Yes & Yes & Yes & 4 & $\begin{array}{l}1342232300 \\
1342232301 \\
\end{array}$ & $\begin{array}{l}2011-11-13 \\
2011-11-13 \\
\end{array}$ \\
\hline NGC 7026 & 316.5773 & 47.8519 & Yes & Yes & No & 3 & $\begin{array}{l}1342234268 \\
1342234269 \\
\end{array}$ & $\begin{array}{l}2011-12-06 \\
2011-12-06 \\
\end{array}$ \\
\hline NML Cyg & 311.606 & 40.1165 & No & Yes & Yes & - & $\begin{array}{l}1342198174 \\
1342198175\end{array}$ & $\begin{array}{l}2010-06-02 \\
2010-06-02 \\
\end{array}$ \\
\hline NML Tau & 58.3701 & 11.4062 & Yes & Yes & Yes & - & $\begin{array}{l}1342203679 \\
1342203680 \\
1342203681 \\
\end{array}$ & $\begin{array}{l}2010-08-28 \\
2010-08-28 \\
2010-08-28 \\
\end{array}$ \\
\hline OH $21.5+0.5$ & 277.1316 & -9.9696 & No & No & No & 2,7 & $\begin{array}{l}1342242438 \\
1342268778 \\
1342268748 \\
\end{array}$ & $\begin{array}{l}2012-03-26 \\
2013-03-29 \\
2013-03-30 \\
\end{array}$ \\
\hline OH $26.5+0.6$ & 279.3852 & -5.3998 & Yes & Yes & Yes & 5 & $\begin{array}{l}1342207776 \\
1342207777\end{array}$ & $\begin{array}{l}2010-10-31 \\
2010-10-31 \\
\end{array}$ \\
\hline OH 30.1-0.7 & 282.1746 & -2.8411 & Yes & Yes & No & - & $\begin{array}{l}1342216207 \\
1342269304 \\
1342269305\end{array}$ & $\begin{array}{l}2011-03-04 \\
2013-04-03 \\
2013-04-03 \\
\end{array}$ \\
\hline OH $30.7+0.4$ & 281.5241 & -1.9881 & Yes & No & No & 6 & $\begin{array}{l}1342268789 \\
1342268790 \\
\end{array}$ & $\begin{array}{l}2013-03-30 \\
2013-03-30 \\
\end{array}$ \\
\hline OH 32.8-0.3 & 283.0924 & -0.2371 & Yes & No & Yes & 5,7 & $\begin{array}{l}1342209738 \\
1342268793 \\
1342268794 \\
\end{array}$ & $\begin{array}{l}2010-11-07 \\
2013-03-30 \\
2013-03-30 \\
\end{array}$ \\
\hline $\mathrm{OH} 104.91+2.41$ & 334.8645 & 59.8560 & Yes & Yes & Yes & 1 & 1342212261 & 2011-01-01 \\
\hline
\end{tabular}

\begin{tabular}{|c|c|c|c|c|c|c|c|c|}
\hline $\mathrm{OH} 231.8+4.2$ & 115.5701 & -14.7144 & Yes & Yes & No & - & $\begin{array}{l}1342196694 \\
1342196695 \\
\end{array}$ & $\begin{array}{l}2010-05-19 \\
2010-05-19 \\
\end{array}$ \\
\hline$o$ Cet & 34.8366 & -2.9776 & Yes & Yes & No & - & $\begin{array}{l}1342213286 \\
1342213287 \\
\end{array}$ & $\begin{array}{l}2011-01-25 \\
2011-01-25 \\
\end{array}$ \\
\hline$\pi \mathrm{Gru}$ & 335.6842 & -45.9479 & Yes & Yes & Yes & - & $\begin{array}{l}1342210397 \\
1342210398\end{array}$ & $\begin{array}{l}2010-11-28 \\
2010-11-28 \\
\end{array}$ \\
\hline R Aql & 286.5927 & 8.2300 & Yes & Yes & Yes & 1,5 & 1342243900 & 2012-04-08 \\
\hline R Cas & 359.6036 & 51.3888 & Yes & Yes & Yes & - & $\begin{array}{l}1342212576 \\
1342212577\end{array}$ & $\begin{array}{l}\text { 2011-01-12 } \\
2011-01-12 \\
\end{array}$ \\
\hline R Dor & 69.1899 & -62.0771 & Yes & Yes & Yes & 3 & $\begin{array}{l}1342197794 \\
1342197795 \\
\end{array}$ & $\begin{array}{l}2010-06-05 \\
2010-06-05 \\
\end{array}$ \\
\hline R Lep & 74.9014 & -14.8062 & Yes & Yes & No & 1,6 & 1342249509 & 2012-08-14 \\
\hline RAFGL 2374 & 290.4021 & 9.4656 & Yes & Yes & No & 1,6 & 1342244920 & 2012-04-24 \\
\hline Red Rectangle & 94.9925 & -10.6374 & Yes & Yes & Yes & - & $\begin{array}{l}1342220928 \\
1342220929\end{array}$ & $\begin{array}{l}2011-04-30 \\
2011-04-30 \\
\end{array}$ \\
\hline RR Aql & 299.4002 & -1.8864 & Yes & Yes & No & 1 & 1342269414 & 2013-04-05 \\
\hline RT Cap & 304.2772 & -21.3179 & Yes & Yes & No & 6 & $\begin{array}{l}1342269308 \\
1342269355\end{array}$ & $\begin{array}{l}2013-04-03 \\
2013-04-04 \\
\end{array}$ \\
\hline S Aur & 81.7810 & 34.1496 & Yes & Yes & No & 1,6 & 1342250896 & 2012-09-11 \\
\hline ST Her & 237.6942 & 48.4830 & Yes & Yes & No & 1,6 & 1342247537 & 2012-06-30 \\
\hline T Cep & 317.3824 & 68.4908 & Yes & Yes & No & 1 & 1342246557 & 2012-06-01 \\
\hline $\mathrm{T}$ Mic & 306.9799 & -28.2610 & Yes & Yes & No & - & $\begin{array}{l}1342268729 \\
1342268788 \\
1342269458\end{array}$ & $\begin{array}{l}2013-03-29 \\
2013-03-30 \\
2013-04-06 \\
\end{array}$ \\
\hline
\end{tabular}


Table A.1. continued.

\begin{tabular}{|c|c|c|c|c|c|c|c|c|}
\hline Target name & RA (deg) & Dec (deg) & IRAS & AKARI & ISO & Comments & ObsIDs & Date \\
\hline Tc 1 & 266.3970 & -46.0899 & Yes & Yes & No & 6 & $\begin{array}{l}1342231319 \\
1342231320 \\
\end{array}$ & $\begin{array}{l}2011-10-22 \\
2011-10-22 \\
\end{array}$ \\
\hline TX Cam & 75.2099 & 56.1812 & Yes & Yes & Yes & 3 & $\begin{array}{l}1342225855 \\
1342225856 \\
\end{array}$ & $\begin{array}{l}2011-08-08 \\
2011-08-08 \\
\end{array}$ \\
\hline U Hya & 159.3886 & -13.3845 & Yes & Yes & No & 1,6 & 1342256947 & 2012-12-11 \\
\hline U Mon & 112.6977 & -9.7768 & Yes & Yes & No & - & $\begin{array}{l}1342206993 \\
1342245243 \\
1342245244 \\
\end{array}$ & $\begin{array}{l}2010-10-23 \\
2012-05-02 \\
2012-05-02 \\
\end{array}$ \\
\hline V384 Per & 51.6229 & 47.5301 & Yes & Yes & Yes & 1,6 & 1342250572 & 2012-09-04 \\
\hline V438 Oph & 258.6657 & 11.0694 & Yes & Yes & No & 1,6 & 1342252326 & 2012-10-07 \\
\hline V1300 Aql & 302.6161 & -6.2704 & Yes & Yes & Yes & - & $\begin{array}{l}1342269516 \\
1342269910 \\
\end{array}$ & $\begin{array}{l}2013-04-07 \\
2013-04-11 \\
\end{array}$ \\
\hline V Cyg & 310.3261 & 48.1413 & Yes & Yes & Yes & 5 & $\begin{array}{l}1342208939 \\
1342208940 \\
\end{array}$ & $\begin{array}{l}2010-11-15 \\
2010-11-15 \\
\end{array}$ \\
\hline V Hya & 162.9052 & -21.2500 & Yes & Yes & Yes & - & $\begin{array}{l}1342197790 \\
1342197791 \\
\end{array}$ & $\begin{array}{l}2010-06-05 \\
2010-06-05 \\
\end{array}$ \\
\hline W Aql & 288.8476 & -7.0471 & Yes & Yes & Yes & - & $\begin{array}{l}1342209731 \\
1342209732 \\
\end{array}$ & $\begin{array}{l}2010-11-06 \\
2010-11-07 \\
\end{array}$ \\
\hline W Hya & 207.2583 & -28.3676 & Yes & Yes & Yes & - & $\begin{array}{l}1342212604 \\
1342223808 \\
\end{array}$ & $\begin{array}{l}\text { 2011-01-14 } \\
2011-07-09 \\
\end{array}$ \\
\hline W Ori & 76.3488 & 1.1776 & Yes & Yes & No & 1,6 & 1342249503 & 2012-08-14 \\
\hline WX Psc & 16.6082 & 12.5980 & Yes & Yes & Yes & - & $\begin{array}{l}1342202121 \\
1342202122 \\
\end{array}$ & $\begin{array}{l}2010-07-28 \\
2010-07-28 \\
\end{array}$ \\
\hline X Her & 240.6632 & 47.2403 & Yes & Yes & No & - & $\begin{array}{l}1342197802 \\
1342197803 \\
1342202120 \\
\end{array}$ & $\begin{array}{l}2010-06-05 \\
2010-06-05 \\
2010-07-28 \\
\end{array}$ \\
\hline $\mathrm{YCVn}$ & 191.2826 & 45.4402 & Yes & Yes & Yes & 1,6 & 1342254305 & 2012-11-02 \\
\hline
\end{tabular}


Table A.2. . Photometric data of THROES targets.

\begin{tabular}{|c|c|c|c|c|c|c|}
\hline Target name & $b(\operatorname{deg})$ & $l(\mathrm{deg})$ & $\operatorname{IRAS}_{100}(\mathrm{Jy})$ & $\operatorname{AKARI}_{160}(\mathrm{Jy})$ & PACS $_{\text {IRAS100 }}(\mathrm{Jy})$ & PACS $_{\text {AKARI160 }}(\mathrm{Jy})$ \\
\hline AC Her & 14.2415 & 50.4928 & 8.0 & $<3.2$ & - & - \\
\hline AFGL 618 & -6.5275 & 166.4460 & 340.0 & 111.7 & 859.0 & 195.9 \\
\hline AFGL 2019 & -0.4324 & 2.5826 & $<227.0$ & 48.6 & - & - \\
\hline AFGL 2403 & 0.7353 & 54.9494 & $<31.3$ & $<10.1$ & - & - \\
\hline AFGL 2513 & -0.8653 & 69.3522 & 20.8 & $<5.8$ & 10.7 & 3.9 \\
\hline AFGL 2688 & -6.5032 & 80.1675 & $<863.0$ & - & 1969.1 & 467.8 \\
\hline AFGL 3068 & -40.3540 & 93.5266 & 73.7 & 24.4 & 102.0 & 34.3 \\
\hline AFGL 3116 & -17.1467 & 108.4549 & 35.5 & 10.9 & 46.8 & 14.9 \\
\hline AFGL 4106 & -1.8789 & 285.1439 & 181.0 & 60.1 & - & - \\
\hline AFGL 4202 & -2.4514 & 316.5887 & $<75.3$ & $<6.5$ & - & - \\
\hline AFGL 4259 & -2.7141 & 65.3167 & 7.5 & $<2.6$ & - & - \\
\hline AFGL 5379 & -1.3330 & 357.3084 & 406.0 & $<31.2$ & - & - \\
\hline AFGL 6815 & 2.9842 & 353.8442 & 82.4 & $<18.37$ & 120.0 & 40.3 \\
\hline AQ Sgr & -16.7007 & 22.7377 & 5.9 & $<0.5$ & 1.3 & 0.4 \\
\hline $\mathrm{BD}+303639$ & 5.0194 & 64.7853 & 70.1 & $<23.1$ & 70.6 & 21.0 \\
\hline CIT 6 & 55.9641 & 197.7148 & 86.1 & - & 83.5 & 24.2 \\
\hline CPD-568032 & -9.9082 & 332.9152 & 91.7 & 29.4 & 106.2 & 26.7 \\
\hline CPD-642939 & -4.2026 & 313.8867 & 20.6 & $<4.7$ & - & - \\
\hline EP Aqr & -39.2603 & 54.2000 & 16.4 & $<4.9$ & 15.0 & 4.7 \\
\hline G Her & 43.7146 & 66.1579 & 6.6 & $<0.1$ & - & - \\
\hline HD 56126 & 9.9947 & 206.7457 & 18.7 & $<3.8$ & 20.5 & 6.0 \\
\hline HD 161796 & 30.8696 & 77.1331 & 48.7 & 10.7 & 56.1 & 8.0 \\
\hline HD 235858 & -2.5181 & 103.3488 & 41.0 & 8.5 & 34.0 & 10.0 \\
\hline HD 331319 & 2.7327 & 67.1633 & 14.8 & $<4.1$ & - & - \\
\hline Hen $2-90$ & 1.4683 & 305.1099 & $<50.2$ & $<11.1$ & - & - \\
\hline Hen 2-113 & 3.9885 & 321.0483 & 71.3 & 20.9 & 90.7 & 26.0 \\
\hline Hen 3-401 & -2.7162 & 285.1194 & 41.5 & 16.6 & 45.2 & 15.7 \\
\hline Hen 3-1475 & 5.7788 & 9.3644 & 33.4 & 12.1 & 36.2 & 9.7 \\
\hline IC 418 & -24.2837 & 215.2120 & 31.2 & - & 29.1 & 9.5 \\
\hline IRAS 07027-7934 & -26.2939 & 291.3749 & 13.6 & $<4.4$ & - & - \\
\hline IRAS 08011-3627 & -2.9959 & 253.0207 & 12.0 & $<4.7$ & 13.6 & 4.6 \\
\hline IRAS 08544-4431 & 0.3864 & 265.5011 & $<28.4$ & $<3.2$ & 29.3 & 11.5 \\
\hline IRAS 09256-6324 & -9.2362 & 282.4206 & 13.5 & $<5.3$ & 15.6 & 7.1 \\
\hline IRAS $09371+1212$ & 42.7271 & 221.8893 & 28.2 & $<11.6$ & 32.7 & 15.5 \\
\hline IRAS 09425-6040 & -5.8842 & 282.0386 & 5.1 & $<1.3$ & 7.5 & 1.8 \\
\hline IRAS 10456-5712 & 1.4921 & 286.8707 & 10.9 & $<5.1$ & 15.6 & 5.8 \\
\hline IRAS 13428-6232 & -0.5939 & 309.1588 & $<186.0$ & 36.9 & - & - \\
\hline IRAS 15194-5115 & 4.6593 & 325.5339 & 51.0 & 15.0 & 37.0 & 11.4 \\
\hline IRAS 16122-5128 & -0.6095 & 331.8689 & $<387.0$ & $<16.1$ & - & - \\
\hline IRAS $16279-4757$ & 0.0851 & 336.1433 & $<265.0$ & - & - & - \\
\hline IRAS 16342-3814 & 5.8464 & 344.0739 & 139.0 & 43.2 & 193.9 & 65.4 \\
\hline IRAS 16594-4656 & -3.2887 & 340.3924 & 34.4 & $<10.6$ & 45.7 & 10.8 \\
\hline IRAS $17010-3840$ & 1.5481 & 347.1050 & $<50.1$ & $<6.3$ & - & - \\
\hline IRAS 17251-2821 & 3.4922 & 358.4142 & $<19.9$ & - & - & - \\
\hline IRAS 17276-2846 & 2.8046 & 358.3664 & $<30.0$ & - & - & - \\
\hline IRAS 17323-2424 & 4.3084 & 2.6112 & $<13.5$ & $<0.7$ & - & - \\
\hline IRAS $17347-3139$ & -0.0557 & 356.8018 & $<249.0$ & - & - & - \\
\hline IRAS 17521-2938 & -2.1916 & 0.4718 & $<110.0$ & - & - & - \\
\hline IRAS $18433-0228$ & 0.1239 & 30.1503 & $<240.0$ & - & - & - \\
\hline IRAS 18488-0107 & -0.4851 & 31.9844 & 72.8 & - & 20.9 & 6.9 \\
\hline IRAS $19067+0811$ & -0.1323 & 42.3093 & $<91.1$ & - & 12.4 & 2.4 \\
\hline IRAS $19306+1407$ & -2.4774 & 50.3034 & 10.0 & $<7.6$ & - & - \\
\hline IRAS 19474-0744 & -16.4859 & 32.7232 & 17.2 & $<3.2$ & 20.8 & 6.5 \\
\hline IRAS $20000+3239$ & 1.1609 & 69.6792 & $<43.1$ & - & 12.0 & 3.4 \\
\hline IRAS 20038-2722 & -27.6822 & 14.7708 & 13.6 & $<0.8$ & 9.8 & 3.3 \\
\hline IRAS $21282+5050$ & -0.1178 & 93.9871 & 15.0 & $<3.7$ & 16.6 & 7.2 \\
\hline IRAS 21554+6204 & 5.9916 & 104.1301 & 15.6 & $<3.3$ & - & - \\
\hline
\end{tabular}

Notes. Target name (Col. 1), as it appears in HSA, galactic coordinates (Cols. 2 and 3), IRAS (100 $\mu \mathrm{m})$ and AKARI (160 $\mu \mathrm{m})$ photometric data (Cols. 4 and 5), and PACS synthetic photometry at 100 and $160 \mu \mathrm{m}$ (Cols. 6 and 7). 
Table A.2. continued.

\begin{tabular}{|c|c|c|c|c|c|c|}
\hline Target name & $b(\mathrm{deg})$ & $l(\mathrm{deg})$ & $\operatorname{IRAS}_{100}(\mathrm{Jy})$ & $\operatorname{AKARI}_{160}(\mathrm{Jy})$ & PACS $_{\text {IRAS100 }}(\mathrm{Jy})$ & PACS $_{\text {AKARI160 }}(\mathrm{Jy})$ \\
\hline IRAS $22036+5306$ & -1.8353 & 99.6333 & 50.7 & 20.3 & 66.1 & 21.2 \\
\hline IRC +10216 & 45.0604 & 221.4467 & 922.0 & - & - & - \\
\hline IRC $-10529^{14}$ & -20.4151 & 36.3565 & 63.7 & 11.4 & - & - \\
\hline IRC +50137 & 7.8349 & 156.4379 & 23.0 & 6.6 & - & - \\
\hline$\chi$ Cyg & 3.2762 & 68.5386 & 17.7 & $<6.9$ & 24.2 & 6.8 \\
\hline MGE 4602 & 0.4734 & 9.3523 & $<50.0$ & $<14.0$ & - & - \\
\hline Mz 3 & -1.0113 & 331.7275 & 113.0 & 24.7 & 77.5 & 28.3 \\
\hline NGC 40 & 9.8680 & 120.0162 & 27.5 & 9.1 & 11.5 & 4.8 \\
\hline NGC 2392 & 17.4002 & 197.8784 & 16.1 & $<3.3$ & - & - \\
\hline NGC 3242 & 32.0509 & 261.0499 & 29.6 & 6.4 & 15.4 & 3.6 \\
\hline NGC 6302 & 1.0558 & 349.5075 & 537.0 & 201.7 & - & - \\
\hline NGC 6445 & 3.9051 & 8.0758 & 43.2 & 14.6 & 13.4 & 3.5 \\
\hline NGC 6537 & 0.7395 & 10.0989 & 166.0 & $<85.5$ & 155.4 & 70.9 \\
\hline NGC 6543 & 29.9548 & 96.4680 & 60.9 & 13.9 & 48.5 & 13.0 \\
\hline NGC 6543 WKnot & 29.9810 & 96.4712 & - & - & - & - \\
\hline NGC 6720 & 13.9782 & 63.1700 & 54.6 & 20.6 & - & - \\
\hline NGC 6720 OFFcenter & 13.9814 & 63.1725 & 54.6 & 20.6 & - & - \\
\hline NGC 6781 & -2.9878 & 41.8407 & 77.0 & - & 0.6 & 1.6 \\
\hline NGC 6781 Rim & -3.0000 & 41.8475 & 77.0 & - & - & - \\
\hline NGC 6826 & 12.7923 & 83.5616 & 21.3 & $<3.7$ & 11.7 & 5.8 \\
\hline NGC 6826 Rim & 12.7828 & 83.5713 & - & - & - & - \\
\hline NGC 7009 & -34.5713 & 37.7621 & 48.1 & 11.3 & 29.0 & 8.6 \\
\hline NGC 7026 & 0.3753 & 89.0020 & 30.9 & $<5.8$ & 17.8 & 1.7 \\
\hline NML Cyg & -1.9208 & 80.7982 & $<334.0$ & 100.1 & 400.0 & 109.2 \\
\hline NML Tau & -31.4120 & 177.9545 & 103.0 & 27.4 & 131.1 & 22.9 \\
\hline OH $21.5+0.5$ & 0.4893 & 21.4588 & $<319.0$ & - & - & - \\
\hline OH $26.5+0.6$ & 0.6179 & 26.5434 & $<311.0$ & $<30.25$ & 181.0 & 33.5 \\
\hline OH 30.1-0.7 & -0.6862 & 30.0911 & $<807.0$ & - & 93.6 & 15.0 \\
\hline $\mathrm{OH} 30.7+0.4$ & 0.2813 & 30.5537 & 290.0 & - & 6.9 & 12.7 \\
\hline OH 32.8-0.3 & -0.3154 & 32.8271 & $<583.0$ & - & 68.6 & 14.0 \\
\hline $\mathrm{OH} 104.91+2.41$ & 2.4134 & 104.9083 & 35.0 & 7.8 & - & - \\
\hline $\mathrm{OH} 231.8+4.2$ & 4.2196 & 231.8354 & 294.0 & 98.9 & 586.2 & 198.0 \\
\hline$o$ Cet & -57.9827 & 167.7549 & 88.4 & 16.1 & 74.3 & 18.5 \\
\hline$\pi \mathrm{Gru}$ & -55.1638 & 350.2822 & 23.3 & $<5.5$ & 18.4 & 6.0 \\
\hline R Aql & 0.4539 & 41.9524 & $<83.1$ & - & - & - \\
\hline R Cas & -10.6191 & 114.5608 & 38.8 & 9.5 & 29.6 & 10.5 \\
\hline R Dor & -39.3424 & 272.6713 & 83.4 & 25.5 & 92.8 & 33.2 \\
\hline R Lep & -31.3270 & 214.3244 & 9.1 & $<0.5$ & - & - \\
\hline RAFGL 2374 & -2.3064 & 44.7947 & 9.8 & $<4.5$ & - & - \\
\hline Red Rectangle & -11.7648 & 218.9680 & 66.2 & $<28.0$ & 88.4 & 35.0 \\
\hline RR Aql & -15.5601 & 38.9171 & 10.1 & $<1.4$ & - & - \\
\hline RT Cap & -27.9386 & 21.9411 & 3.6 & $<3.8$ & 2.2 & 0.6 \\
\hline S Aur & -0.5112 & 173.4866 & 11.6 & $<0.7$ & - & - \\
\hline ST Her & 49.4432 & 76.9788 & 6.0 & $<1.4$ & - & - \\
\hline T Cep & 13.8449 & 104.8050 & 15.3 & $<3.0$ & - & - \\
\hline T Mic & -32.4287 & 15.1857 & 12.7 & $<3.1$ & 10.4 & 3.2 \\
\hline Tc 1 & -8.8349 & 345.2375 & 4.7 & $<0.5$ & 2.0 & 1.0 \\
\hline U Hya & 38.0741 & 259.9663 & 14.5 & $<0.8$ & - & - \\
\hline U Mon & 4.1536 & 226.1413 & 9.5 & - & 12.0 & 4.9 \\
\hline V384 Per & -7.5975 & 148.1771 & 11.9 & - & - & - \\
\hline V438 Oph & 26.4554 & 32.1306 & 3.7 & - & - & - \\
\hline V1300 Aql & -20.4165 & 36.3580 & 63.7 & 11.4 & 72.7 & 17.5 \\
\hline V Cyg & 3.7667 & 86.5361 & 17.2 & $<6.0$ & 13.3 & 5.2 \\
\hline V Hya & 33.6014 & 268.9648 & 29.9 & 8.0 & 33.9 & 9.6 \\
\hline W Aql & -8.5161 & 29.3389 & 36.0 & 10.6 & 26.6 & 7.6 \\
\hline W Hya & 32.8108 & 318.0223 & 72.2 & 24.3 & 72.3 & 26.6 \\
\hline W Ori & -22.8179 & 199.0092 & 6.27 & $<1.8$ & - & - \\
\hline WX Psc & -50.1074 & 128.6416 & 72.1 & $<15.0$ & 77.5 & 19.8 \\
\hline X Her & 47.7855 & 74.4639 & 18.3 & $<3.3$ & 9.5 & 3.6 \\
\hline Y CVn & 71.6450 & 126.4472 & 7.8 & - & - & - \\
\hline
\end{tabular}

